\title{
PULSARS AND GRAVITY
}

\author{
R. N. Manchester \\ CSIRO Astronomy and Space Science \\ Epping NSW 1710, Australia \\ dick.manchester@csiro.au
}

\begin{abstract}
Pulsars are wonderful gravitational probes. Their tiny size and stellar mass give their rotation periods a stability comparable to that of atomic frequency standards. This is especially true of the rapidly rotating "millisecond pulsars" (MSPs). Many of these rapidly rotating pulsars are in orbit with another star, allowing pulsar timing to probe relativistic perturbations to the orbital motion. Pulsars have provided the most stringent tests of theories of relativistic gravitation, especially in the strong-field regime, and have shown that Einstein's general theory of relativity is an accurate description of the observed motions. Many other gravitational theories are effectively ruled out or at least severely constrained by these results. MSPs can also be used to form a "Pulsar Timing Array" (PTA). PTAs are Galactic-scale interferometers that have the potential to directly detect nanohertz gravitational waves from astrophysical sources. Orbiting super-massive black holes in the cores of distant galaxies are the sources most likely to be detectable. Although no evidence for gravitational waves has yet been found in PTA data sets, the latest limits are seriously constraining current ideas on galaxy and black-hole evolution in the early Universe.
\end{abstract}

Keywords: gravity — gravitational waves — pulsars — pulsar timing — general relativity

\section{Introduction}

Pulsars are rotating neutron stars that emit beams of radiation which sweep across the sky as the star rotates. A beam sweeping across the Earth may be detected as a pulse that repeats with a periodicity equal to the rotation period of the star. Because of the large mass of neutron stars, $\sim 1.4 \mathrm{M}_{\odot}$, and their tiny size, radii $\sim 15 \mathrm{~km}$, (see Ref. 76 for a review of neutron-star properties) the rotation period of neutron stars is incredibly stable, with a stability comparable to that of the best atomic clocks on Earth. This great period stability, combined with the fact that pulsars are often in a binary orbit with another star, makes them wonderful probes of relativistic gravity. Tiny perturbations to their period resulting from, for example, relativistic effects in a binary orbit, may be detected and compared with the predictions of a gravitational theory. Pulsars may also be used as detectors for gravitational waves passing through the Galaxy. To separate the effects of gravitational waves from other perturbations, signals from pulsars in different directions on the sky must be compared - exactly analogous to the way laser-interferometer gravitational-wave detectors compare laser phases in perpendicular arms. 
More than 2400 pulsars are now known The vast majority of them reside in our Galaxy, typically at distances of a few thousand light-years from the Sun. Their pulse periods, $P$, range between 1.4 milliseconds and 12 seconds and fall into two main groups. The so-called "normal" pulsars have periods longer than about 30 milliseconds and the "millisecond" pulsars (MSPs) have shorter periods. MSPs comprise about $15 \%$ of the known pulsar population.

Although pulsar periods are very stable, they are not constant. In their own reference frame, all pulsars are slowing down, albeit very slowly. Pulsars are powered by their rotational energy. They have extremely strong magnetic fields and, as they spin, they emit streams of relativistic particles and so-called "dipole radiation", electromagnetic waves with period equal to the rotation period of the star. These carry energy away from the star resulting in a steady increase in the pulse period. Typical rates of period increase, $\dot{P}$, are a part in $10^{15}$ for normal pulsars and much less for MSPs. Assuming that the surrounding magnetic field is predominantly dipole, the characteristic age $\tau_{c}$ of a pulsar is given by

$$
\tau_{c}=P /(2 \dot{P})
$$

and their surface dipole magnetic field strength (in gaus: b) is

$$
B_{s} \approx 3.2 \times 10^{19}(P \dot{P})^{1 / 2} \mathrm{G} \text {. }
$$

Figure 1 shows the distribution of pulsars on the $P-\dot{P}$ plane, with several different types of pulsars indicated. For most normal pulsars, $\tau_{c}$ is between $10^{3}$ and $10^{7}$ years and $B_{s}$ is between $10^{11}$ and $10^{13} \mathrm{G}$. For MSPs, the corresponding ranges are $10^{9}$ to $10^{11}$ yrs and $10^{8}$ to $10^{10} \mathrm{G}$.

Normal pulsars and MSPs have quite different evolutionary histories. Most if not all normal pulsars are formed in supernova explosions at the death of a massive star. They age with relatively constant $B_{s}$ until the pulse emission mechanism begins to fail when $\tau_{c}$ reaches about $10^{6}$ yrs. Many young pulsars $\left(\tau_{c} \lesssim 10^{4} \mathrm{yrs}\right)$ are located within supernova remnants, with the most famous example being the Crab pulsar, PSR B0531+21, located near the centre of the Crab Nebula. Most young pulsars lie relatively close to the Galactic Plane, consistent with the idea that they are formed from massive stars. MSPs on the other hand are much more widely distributed in the Galactic halo. They are believed to originate from old, slowly rotating and probably dead neutron stars that accrete matter and angular momentum from an evolving binary companion. This "recycling" process increases their spin rate so that they have periods in the millisecond range and re-energises the beamed emission (see, e.g., Ref. 17).

Figure 1 shows that the majority of MSPs are members of a binary system, consistent with this formation scenario. The accretion also suppresses their apparent magnetic field, so that MSPs have $\dot{P}$ values about five orders of magnitude less than

aSee the ATNF Pulsar Catalogue: http://www.atnf.csiro.au/research/pulsar/psrcat

${ }^{\mathrm{b}} 1$ gauss $(\mathrm{G})=10^{-4}$ tesla 
normal pulsars. Since the level of intrinsic period irregularities is related to $\dot{P}, 117$ it is this low $B_{s}$ that makes MSPs extremely stable clocks and suitable tools for the study of relativistic gravitation. Figure 1 also indicates the class of radio-quiet pulsars. Essentially all of the 72 known radio-quiet pulsars are young and solitary. They include the so-called "magnetars" which lie in the upper right side of the $P$ $\dot{P}$ diagram where dipole magnetic fields are strongest.

Most double-neutron-star systems lie in the zone between the MSPs and the normal pulsars. These systems are believed to have been partially recycled prior to the formation of the second-born neutron star. In almost every case, the ob-

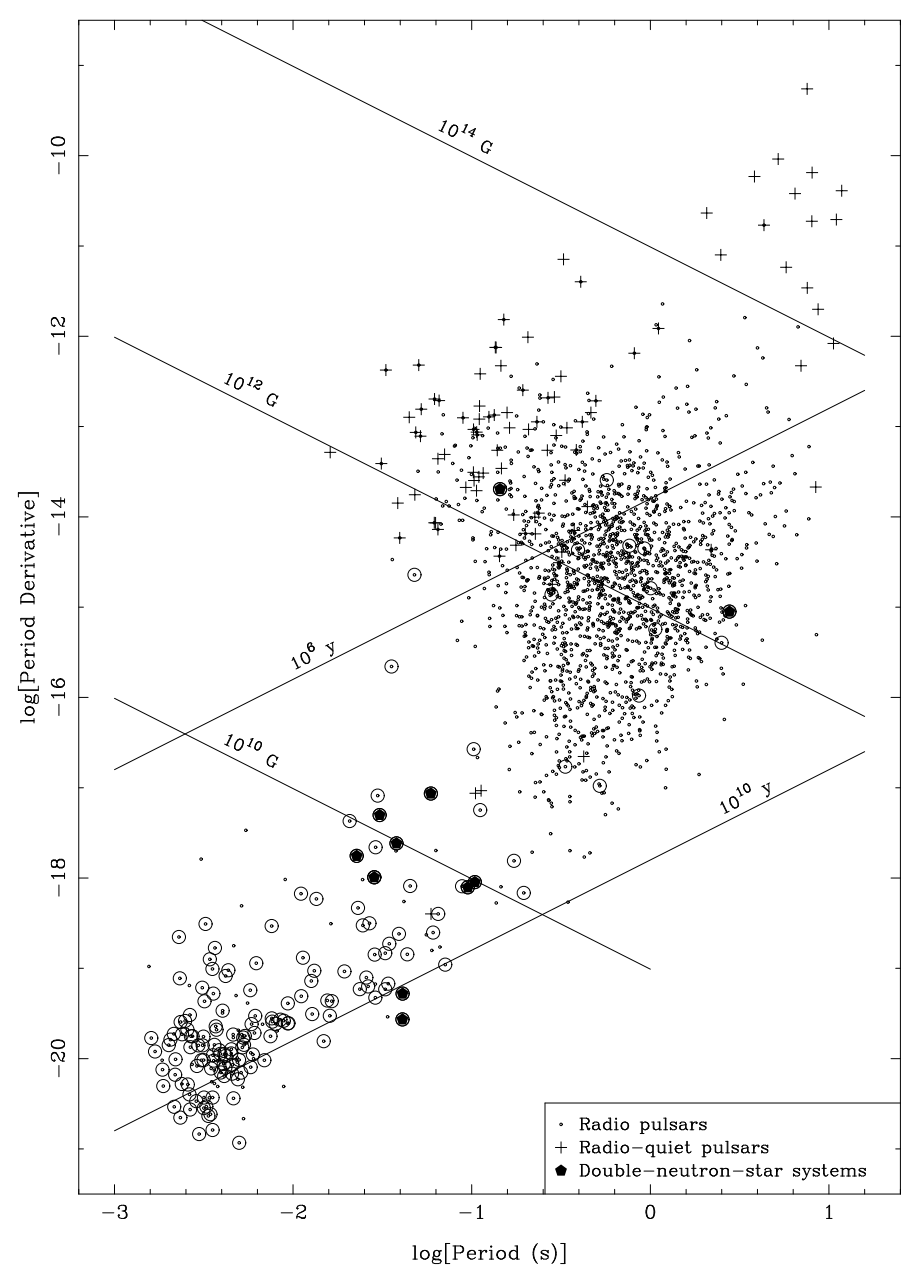

Fig. 1. Distribution of pulsars on the $P-\dot{P}$ plane with radio-loud and radio-quiet pulsars indicated. Binary pulsars are indicated by a circle around the point and, for those with a neutron-star companion, the circle is filled in. Lines of constant characteristic age $\left(\tau_{c}\right)$ and surface-dipole magnetic field $\left(B_{s}\right)$ are shown. 
served pulsar is the recycled one since it has a much longer active lifetime than the newly formed young pulsar. A famous exception to this is the Double Pulsar (PSR J0737-3039A/B) in which the second-born star (B) is (or was) still visible ${ }^{83}$ On Figure 1 the B pulsar is the solitary double-neutron-star system on the right side of the plot. The double-neutron-star system identified near the middle of the plot contains the relatively young pulsar, PSR J1906+0746. There is some doubt about the nature of the companion in this system - it could be a heavy white dwarf 139 These double-neutron-star systems and their use as probes of relativistic gravity are discussed in some detail in Section 2.1 below.

\subsection{Pulsar Timing}

The most important contributions of pulsars to investigations of gravitational theories and gravitational waves rely on precision pulsar timing observations. These allow both the relativistic perturbations to binary orbits to be studied in detail and the potential detection of the tiny period fluctuations generated by gravitational waves passing through our Galaxy. Because of the importance of pulsar timing to these studies, we give here a brief review of its basic principles.

The basic observable in pulsar timing is the time of arrival (ToA) of a pulse at an observatory. In fact, because of signal/noise limitations and the intrinsic fluctuation of individual pulse shapes, ToA measurements are based on mean pulse profiles formed by synchronously averaging the data, typically for times between several minutes and an hour. The time at which a fiducial pulse phase (usually near the pulse peak) arrives at the telescope is determined by cross-correlating the observed mean pulse profile with a standard pulse template. A series of these ToAs is measured over many days, months, years and even decades for the pulsar of interest.

These observatory ToAs are affected by the rotational and orbital motion of the Earth (and for satellite observatories, the orbital motion of the satellite). To remove these effects, the observed ToAs are referred to the solar-system barycentre (centre of mass) which is assumed to be inertial (unaccelerated) with respect to the distant Universe 0 This correction makes use of a solar-system ephemeris giving predictions of the position of the centre of the Earth with respect to the solarsystem barycentre. Such ephemerides, for example, the Jet Propulsion Laboratory ephemeris DE $421, \frac{48}{4}$ are generated by fitting relativistic models to planetary and spacecraft data. The correction also takes into account the relativistic variations in terrestrial time resulting from the Earth's motion.

The resulting barycentric ToAs are then compared with predicted pulse ToAs based on a model for the pulsar. The pulsar model can have 20 or more parameters; generally included are the pulse frequency $(\nu=1 / P)$, frequency time derivative $(\dot{\nu})$, the pulsar position and the five Keplerian binary parameters if the pulsar is a

\footnotetext{
${ }^{\mathrm{c}}$ This neglects any acceleration of the solar-system barycentre resulting from, for example, Galactic rotation. For some precision timing experiments, such effects are taken into account at a later stage of the analysis.
} 
member of a binary system. If the data are recorded at different radio frequencies, it is also necessary to include the dispersion measure (DM) which quantifies the frequency-dependent delay suffered by the pulses as they propagate through the interstellar medium.

The differences between the observed and predicted ToAs are known as "timing residuals". Errors in any of the model parameters result in systematic variations in the timing residuals as a function of time. For example, if the model pulse frequency is too small, the residuals will grow linearly as illustrated in Figure 2 The required correction to the pulse frequency is given by the slope of this variation. An error in the pulsar position results in an annual sine curve which arises from the barycentric correction. The phase and amplitude of this curve give the corrections to the two position coordinates. Similarly, a pulsar proper motion results in a linearly growing sine curve (away from the reference epoch). For a sufficiently close pulsar, the curvature of the wavefront results in a biannual term in the residuals, and offsets in binary parameters result in terms which vary with the orbital periodicity.

Since offsets in different parameters in general result in different systematic residual variations, it is possible to do a least-squares fit to the observed residuals for the correction to any desired parameter The precision of the fitted parameters is often amazingly high. For example, the relative precision of the pulse frequency determination is $\sim \delta t / T$, where $\delta t$ is the typical uncertainty in the ToAs and $T$ is the data span. Since, for MSPs at least, $\delta t$ is often $<1 \mu$ s and $T$ is often many years, the relative precision of the measured $\nu$ can easily exceed $1: 10^{14}$. Similarly pulsar positions can be measured to micro-arcseconds and binary eccentricities measured to $1: 10^{8}$. These very high precisions often allow higher-order terms, for example, resulting from relativistic perturbations to the binary orbit, to be measured.

\section{Tests of Relativistic Gravity}

\subsection{Tests of General Relativity with Double-Neutron-Star Systems}

\subsubsection{The Hulse-Taylor binary, PSR B1913+16}

The discovery at Arecibo Observatory in 1974 of the first-known binary pulsar, PSR B1913+16, by Hulse and Taylor ${ }^{61}$ was remarkable in a number of respects. Firstly, it showed that pulsars with short pulse periods but large characteristic ages (59 $\mathrm{ms}$ and $10^{8} \mathrm{yr}$, respectively, for PSR B1913+16) could exist. The period of PSR B1913+16 was second only to the Crab pulsar, but its age was enormously greater than that of other short-period pulsars known at the time. Secondly, it was in a binary orbit with a relatively massive star, very likely another neutron star, showing that an evolutionary pathway to such systems existed. Thirdly, its orbital period was extraordinarily short, only $7.75 \mathrm{~h}$, its eccentricity large, $\sim 0.617$, and, as shown in Figure 3 its maximum orbital velocity very high, $\sim 300 \mathrm{~km} \mathrm{~s}^{-1}$ or

${ }_{\mathrm{d}}$ The data span must be sufficiently long to avoid excessive covariance between the variations for different fitted parameters. 


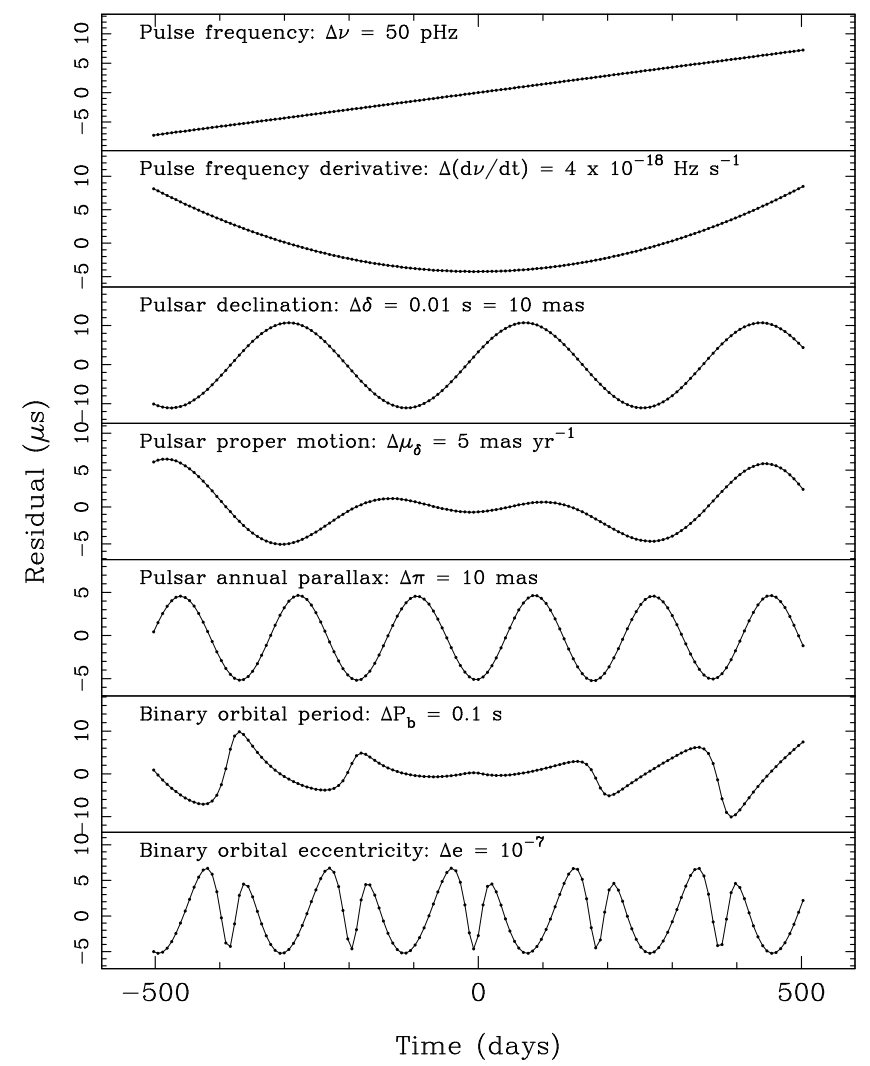

Fig. 2. Variations in timing residuals for offsets in several parameters. The model pulsar has a pulse frequency of $300 \mathrm{~Hz}(3.3 \mathrm{~ms}$ period $)$ and is in an eccentric $(e=0.4)$ binary orbit of period 190 days. The reference epochs for period, position and binary phase are at the middle of the plotted range.

$0.1 \%$ of the velocity of light. As was immediately recognised by Hulse and Taylor, these properties opened up the possibility that relativistic perturbations to the orbit were potentially measurable. Lowest-order relativistic effects go as $(v / c)^{2}$, and so the variations are of order 1:106 , enormous by the standards of pulsar measurements.

Relativistic effects in binary motion can be expressed in terms of "postKeplerian" parameters that describe departures from Keplerian motion (see, e.g., Ref. 129). The first such parameter to be observed was periastron precession! In Einstein's general theory of relativity (GR) the rate of periastron precession (averaged over the binary orbit) is given by:

$$
\dot{\omega}=3\left(P_{b} / 2 \pi\right)^{-5 / 3}\left(T_{\odot} M\right)^{2 / 3}\left(1-e^{2}\right)^{-1}
$$

where $\omega$ is the longitude of periastron measured from the ascending node, $P_{b}$ is the orbital period, $T_{\odot} \equiv G M_{\odot} / c^{3}=4.925490947 \mu \mathrm{s}, G$ is the Gravitational Constant, $M_{\odot}$ is the mass of the Sun, $e$ is the orbital eccentricity and $M=m_{1}+m_{2}$, the sum of the pulsar mass $m_{1}$ and the companion mass $m_{2}$ in solar units. It is worth 


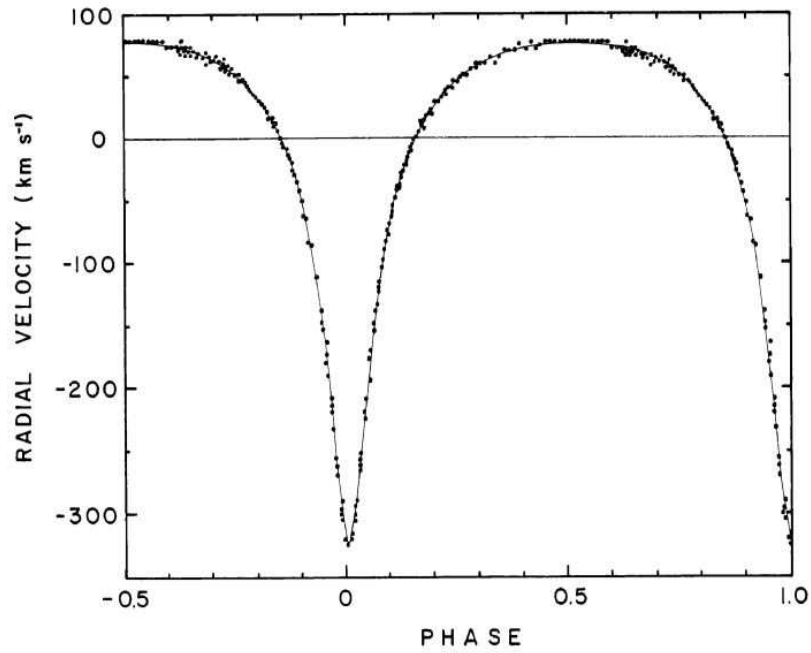

Fig. 3. Velocity curve for the Hulse-Taylor binary pulsar, PSR B1913+16. (Ref. 61)

noting that this is the same effect as the excess perihelion advance of Mercury that was used by Einstein $\frac{43}{3}$ as an observational verification of GR. The relativistic effect for Mercury is just 43 arcsec per century, minuscule compared to the 4.22 per year predicted and observed for PSR B1913+16.

The next most significant parameter, normally labelled $\gamma$, describes the combination of gravitational redshift and 2nd-order (or transverse) Doppler shift, both of which have the same dependence on orbital phase. In GR

$$
\gamma=e\left(P_{b} / 2 \pi\right)^{1 / 3} T_{\odot}^{2 / 3} M^{-4 / 3} m_{2}\left(m_{1}+2 m_{2}\right) .
$$

Since the Keplerian parameters are very well determined, measurement of $\dot{\omega}$ and $\gamma$ gives two equations in two unknowns, $m_{1}$ and $m_{2}$, and so the two stellar masses can be determined. For PSR B1913+16, these are both close to $1.4 M_{\odot}$, confirming the double-neutron-star nature of the system. An important consequence of this was that the two stars could safely be treated as point masses in GR, thereby allowing precise tests of the theory.

Given the Keplerian parameters and the two masses, the next post-Keplerian parameter, orbital decay due to the emission of gravitational waves from the system, given in GR by

$$
\dot{P}_{b}=-\frac{192 \pi}{5}\left(\frac{P_{b}}{2 \pi}\right)^{-5 / 3}\left(1+\frac{73}{24} e^{2}+\frac{37}{96} e^{4}\right)\left(1-e^{2}\right)^{-7 / 2} T_{\odot}^{5 / 3} m_{1} m_{2} M^{-1 / 3},
$$

could be predicted and compared with observation. This therefore constituted a unique test of GR in strong-field gravity. After just four years, the $\dot{P}_{b}$ term was well measured and shown to be consistent with the GR prediction!134 The orbital decay, including more recent results, is illustrated in Figure 4, the ratio of observed to predicted orbit decay is $0.997 \pm 0.002 \frac{143}{{ }^{143}}$ It should be mentioned that this near-perfect 
agreement relies on correcting the observed $\dot{P}_{b}$ for the differential acceleration of the solar system and the pulsar system in the gravitational field of the Galaxy. This correction is $-0.027 \pm 0.005 \times 10^{-12}$ or about $1 \%$ of the observed value, and the uncertainty in the final result is dominated by the uncertainty in this correction. Unfortunately, it is unlikely that this uncertainty will be significantly reduced in the near future since it mainly depends on the poorly known distance to the binary system.

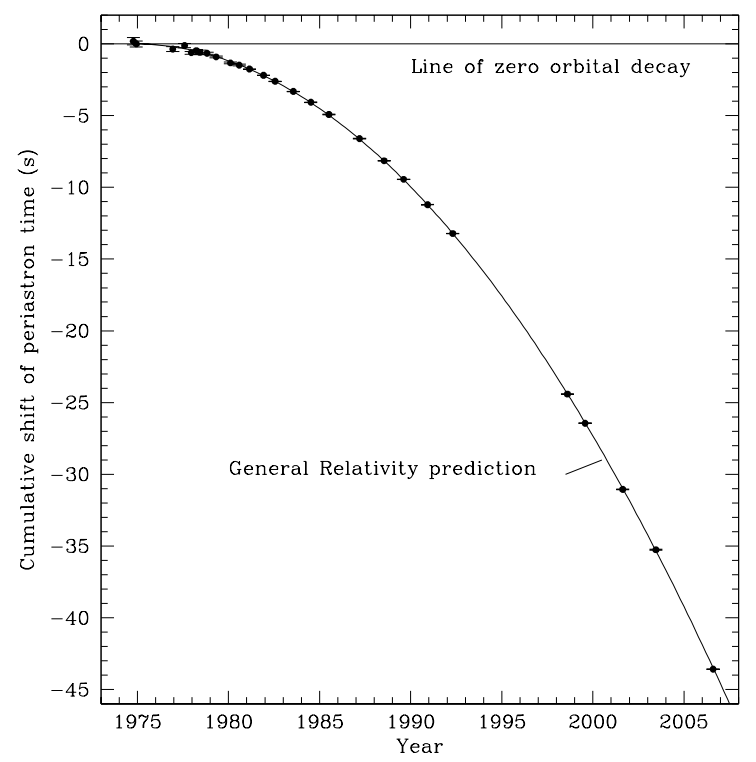

Fig. 4. Comparison of the observed and predicted orbital period decay in PSR B1913+16. The orbital decay is quantified by the shift in the time of periastron passage with respect to a nondecaying orbit. The parabolic curve is the predicted decay from GR. (Ref. 143)

Two more post-Keplerian parameters, denoted by $r$ and $s$, relate to the Shapiro delay suffered by the pulsar signal while passing through the curved spacetime surrounding the companion star. $\frac{123}{123}$ The relations for them in GR are as follows:

$$
\begin{gathered}
r=T_{\odot} m_{2} \\
s \equiv \sin i=\left(a_{1} / c\right) \sin i\left(\frac{P_{b}}{2 \pi}\right)^{-2 / 3} T_{\odot}^{-1 / 3} M^{2 / 3} m_{2}^{-1}
\end{gathered}
$$

where $\left(a_{1} / c\right) \sin i$ is the projected semi-major axis of the pulsar orbit (in time units), a Keplerian parameter. The Shapiro delay is generally only observable when the orbital inclination is relatively close to $90^{\circ}$, that is, the orbit is seen close to edgeon. For the Hulse-Taylor binary pulsar the orbital inclination is about $47^{\circ}$ and the 
Shapiro delay is small and covariant with Keplerian parameters. However it has been observed in a number of other neutron-star binary systems as will be discussed below.

Another relativistic effect, geodetic precession, has observable consequences for PSR B1913+16 and several other binary pulsars. A neutron star formed in a supernova explosion receives a "kick" during or shortly after the explosion which typically gives the star a velocity of several hundred $\mathrm{km} \mathrm{s}^{-1}[59]$ If the pulsar is a member of a binary system which is not disrupted by the kick, the orbital axis is changed so that pulsar spin axis, which was most likely aligned with the orbital axis prior to the explosion, is no longer aligned. Since kick velocities are often comparable to or even larger than the orbital velocities, the misalignment angle can be large. Precession of the spin axis will therefore alter the aspect of the radio beam seen by an observer and may even move the beam out of the line of sight.

In GR, the precessional angular frequency is given by

$$
\Omega_{p}=\frac{1}{2}\left(\frac{P_{b}}{2 \pi}\right)^{-5 / 3} T_{\odot}^{2 / 3} \frac{m_{2}\left(4 m_{1}+3 m_{2}\right)}{\left(1-e^{2}\right) M^{4 / 3}} .
$$

For PSR B1913+16, the corresponding precessional period is about 297 years, so the aspect changes over observational data spans are small. Never-the-less changes in the relative amplitude of the two peaks in the PSR B1913+16 profile were reported by Weisberg et al 144 and attributed to the effects of geodetic precession with a "patchy" beam. For a basically conal beam geometry, the separation of the two profile components would be expected to change and evidence for this was first found by Kramer ${ }^{72}$ leading to an estimate of the misalignment angle of about $22^{\circ}$. A data set extending to 2001 was analysed by Weisberg and Taylor, 145 obtaining results similar to those of Kramer ${ }^{72}$ Their best-fitting model gives the "peanut" shaped beam shown in Figure 5. Clifton and Weisberg ${ }^{28}$ have shown that a set of circular nested emission cones can also give apparent pulse-width variations similar to those observed. Over the 20-year interval covered by the data set, the "impact parameter" (minimum angle between the beam symmetry axis and the observer's line of sight) has changed by a rather small amount, about $3^{\circ}$, from -3.5 to -6.5 . Extrapolation of this model suggests that the pulsar will become unobservable in about 2025. While this result is compatible with relativistic precession, it is not possible to derive an independent measure of the precessional rate from these data.

\subsubsection{PSR B1534+12}

PSR B1534+12, discovered by Wolszczan in $1990, \frac{152}{15}$ a binary system with parameters quite similar to those of B1913+16, notably a short orbital period $(\sim 10.1$ hours $)$, relatively high eccentricity $(\sim 0.27)$ and a compact orbit about $60 \%$ larger than that of PSR B1913+16. Analysis of less than a year's data already allowed measurement of two post-Keplerian parameters, $\dot{\omega}$ and $\gamma$, thereby 


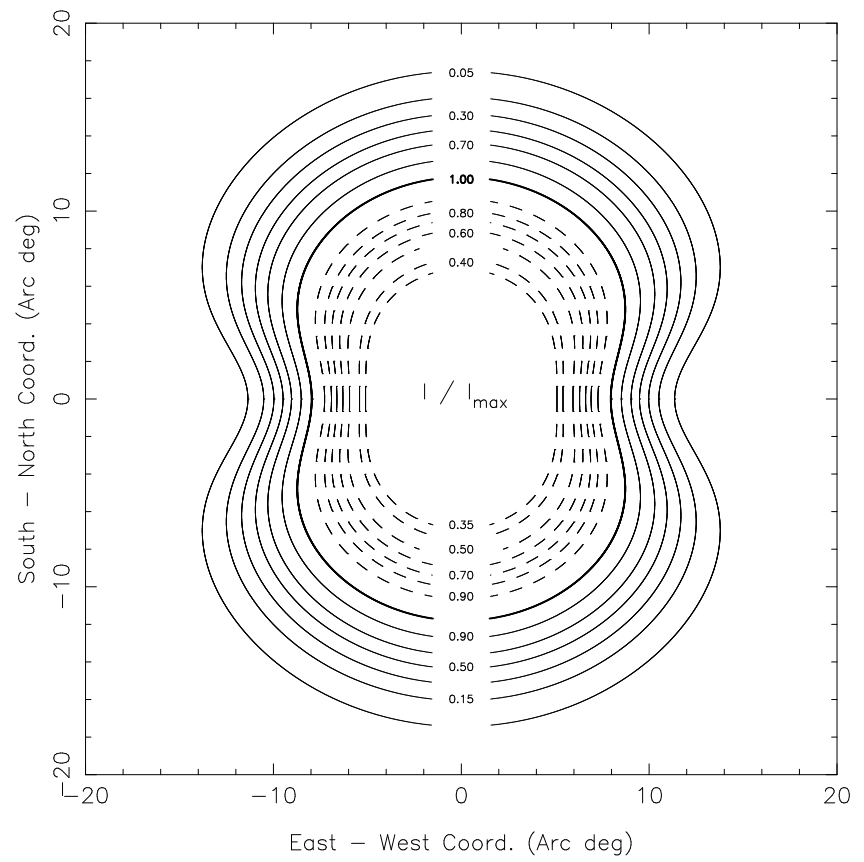

Fig. 5. Contours of the symmetric part of the radio emission beam for PSR B1913+16, obtained by fitting to the variation in pulse width over the interval 1981 to 2001 . (Ref. [145)

determining the masses of the two stars and confirming that they are both neutron stars. These results also showed that the orbit was more edge-on than that of PSR B1913+16, with an implied inclination angle of about $77^{\circ}$. Analysis of timing data extending over 22 years by Fonseca et al $\stackrel{49}{[4}$ built on earlier results by Stairs et al.,131 with significant detections of $r$ and $s$, the Shapiro-delay parameters, and the orbital decay, $\dot{P}_{b}$, giving five post-Keplerian parameters and three independent tests of GR. A fourth test of GR, albeit less precise, was provided by an analysis of the evolution of the profile shape and polarisation, yielding a rate for the geodetic precession of the pulsar spin axis $\Omega_{p}=0.59_{0.08}^{+0.12} \mathrm{yr}^{-1}$ which is consistent with the value predicted by GR. Figure 6 shows the so-called "mass-mass" diagram for PSR B1534+12, illustrating these constraints.

If GR gives a correct description of the post-Keplerian parameters, all of these constraints should be consistent with an allowed range of $m_{1}$ and $m_{2}$. For PSR B1534+12, the masses are most accurately constrained by $\dot{\omega}$ and $\gamma$ but the predicted constraint on $\dot{P}_{b}$ appears to be inconsistent. As for PSR B1913+16, the measured value of $\dot{P}_{b}$ must be corrected for kinematic effects resulting from the differential acceleration of the binary and solar system in the Galaxy. PSR B1534+12 is much closer to the Sun that PSR B1913+16 and the so-called "Shklovskii" effect, 125 an apparent radial acceleration resulting from transverse motion of the binary system $\left(\dot{P} / P=\mu^{2} d / c\right.$, where $\mu$ is the pulsar proper motion and $d$ is the pulsar distance), 
is also important. The distance estimate is based on the pulsar DM and a model for the free electron density in the Galaxy and is quite uncertain. Stairs and her

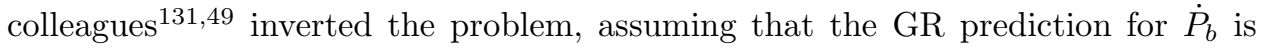
correct, thereby deriving an improved value for the pulsar distance, a technique first suggested by Bell and Bailes 12

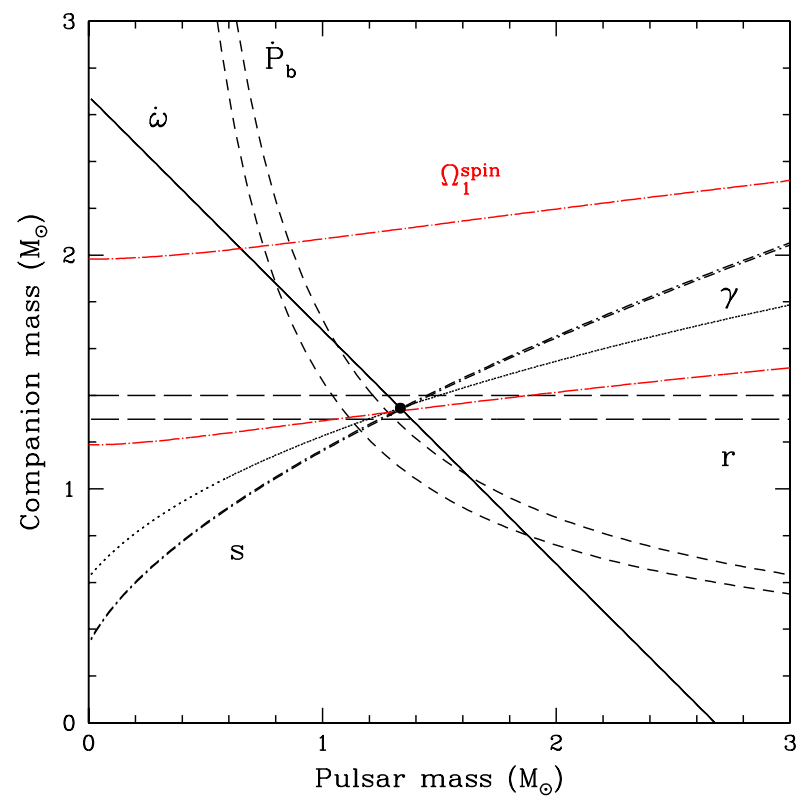

Fig. 6. Plot of companion mass $\left(m_{2}\right)$ versus pulsar mass $\left(m_{1}\right)$ for PSR B1534+12. Constraints derived from the measured post-Keplerian parameters assuming GR are shown pairs of lines with separation indicating the uncertainty range. For $\dot{\omega}$ and $\gamma$, the uncertainty ranges are too small to see on the plot. In addition, a constraint from the measured spin precession rate is shown. (Ref.49)

\subsubsection{The Double Pulsar, PSR J0737-3039A/B}

The discovery of the Double Pulsar system ${ }^{2183}$ heralded a remarkable era for investigation of relativistic effects in double-neutron-star systems. In this system, the A pulsar was formed first and subsequently spun up to approximately its current period of $23 \mathrm{~ms}$ by accretion from its evolving binary companion. The companion then imploded to form the B pulsar which has since spun down to its current period of about $2.8 \mathrm{~s}$. The orbital period is only 2.5 hours, less than a third of that for PSR B1913+16, and the projected semi-major axis $a_{1} \sin i$ is about $60 \%$ that of PSR B1913+16. These parameters imply relativistic effects much larger than those seen for PSR B1913+16; for example, the predicted relativistic periastron advance is $16.9 \mathrm{yr}^{-1}$, more than four times the value for PSR B1913+16. Added to that, the 
orbit is seen within a degree or so of edge-on, not only allowing detailed measurement of the Shapiro delay, but also resulting in eclipses of the A pulsar emission by the magnetosphere of the B pulsar. Finally, the still-unique detection of the second neutron star as a pulsar allows a direct measurement of the mass ratio of the two stars from the ratio of the two non-relativistic Roemer delays $\left(a_{1} \sin i\right)$ @

Four post-Keplerian parameters $(\dot{\omega}, \gamma, r$ and $s)$ were detected in just seven months of timing data from the Parkes $64-\mathrm{m}$ and Lovell $76-\mathrm{m}$ Telescopes ${ }^{83}$ Further observations, including data from the Green Bank 100-m Telescope, with a 2.5-year data span give the currently most stringent test of GR in strong-field conditions. ${ }^{74 / 75}$ Figure 8 shows the mass-mass diagram based on these results together with the measurement of geodetic spin precession for the B pulsar described below. A total of six post-Keplerian parameters together with the mass ratio $R$ gives five independent tests of GR. As well as the three post-Keplerian parameters, $\dot{\omega}, \gamma$ and $\dot{P}_{b}$ observed for PSRs B1913+16 and PSR B1534+12 (Figure 6), for the Double Pulsar we have the mass ratio $R$, the Shapiro delay parameters $r$ and $s$ and a measurement of the rate of geodetic precession $\Omega_{p}$. The observed Shapiro delay (Figure 7) shows that the $\mathrm{J} 0737-3039 \mathrm{~A} / \mathrm{B}$ orbit inclination angle is $88^{\circ} .7 \pm 0.7$.

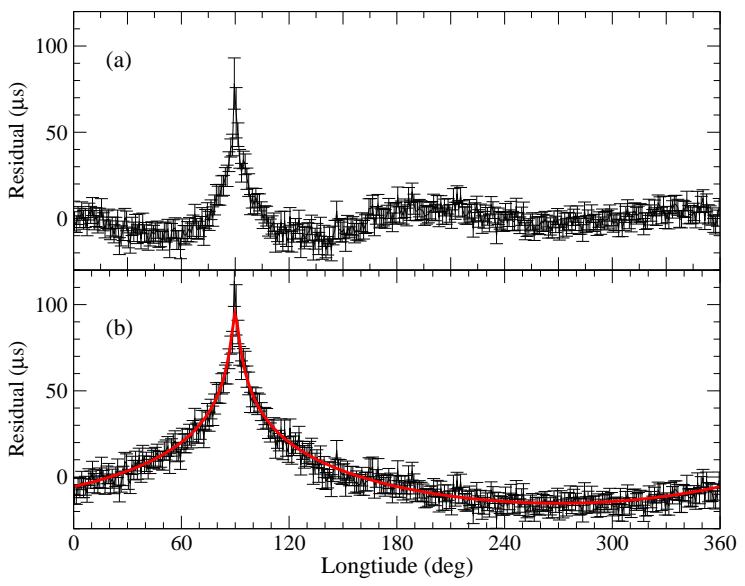

Fig. 7. Observed Shapiro delay as a function of orbital phase for PSR J0737-3039A. Upper panel: timing residuals after fitting for all parameters except the Shapiro-delay terms, $r$ and $s$, with these set to zero. Lower panel: the full Shapiro delay obtained by taking the best-fit values from a full solution, but setting $r$ and $s$ to zero. The red line is the prediction based on GR. (Ref. 74)

As mentioned above, this nearly edge-on view of the orbit results in eclipses of of the A-pulsar emission by the magnetosphere of the B pulsar. These eclipses

eThe B pulsar became undetectable as a radio pulsar in 2008, most probably because the beam precessed out of our line of sight ${ }^{[01}$ Because of uncertainty about the beam shape, the date of its return to visibility is very uncertain, but it should be before 2035 . 
last only about $30 \mathrm{~s}$, showing that the B-pulsar magnetosphere is highly modified by the relativistic wind from pulsar $\mathrm{A}{ }^{[83}$ Remarkably, observations with high time resolution made with the Green Bank Telescope showed that the eclipse is modulated at the spin period of pulsar B.$^{88}$ Modelling of the detailed eclipse profile by absorption in the doughnut-shaped closed-field-line region of the magnetosphere by Lyutikov and Thompson ${ }^{84}$ allowed determination of the geometry of the binary system including the offset between the B-pulsar spin axis and the orbital angular momentum axis, the so-called "misalignment angle" which they estimate to be about $60^{\circ}$. Even more remarkably, detailed measurements of the eclipse profile over about four years enabled Breton et al ${ }^{20}$ to directly estimate the rate of geodetic precession as $4.77 \pm 0.66 \mathrm{yr}^{-1}$, consistent with the predicted precessional period of 70.95 years based on GR (Equation 8). It is notable that no secular profile evolution has been observed for PSR J0737-3039A, implying that, unlike for the B pulsar, the misalignment angle for pulsar A is very small! 45

The most precisely measured post-Keplerian parameter is $\dot{\omega}$. This constraint is nearly orthogonal to that from the mass ratio $R$ (Figure 8 ) giving values for the two neutron-star masses of $m_{1}=1.3381 \pm 0.0007 \mathrm{M}_{\odot}$ and $m_{2}=1.2849 \pm 0.0007 \mathrm{M}_{\odot} .{ }^{74}$ Together with the accurately measured Keplerian parameters, these two masses can be used to predict the remaining five post-Keplerian parameters using Equations 4 -8. As Figure 8 shows, GR gives a self-consistent description of the orbital motions, with all five independent constraints consistent with the masses derived from $\dot{\omega}$ and $R$.

The most stringent test comes from the derived value of $s, 0.99974 \pm 0.00039$. The ratio of the observed and predicted values of $s$ is $0.99987 \pm 0.0005$, by far the most constraining test of GR in the strong-field regime. Furthermore, this test is qualitatively different to that for PSR B1913+16 in that it is based on a nonradiative prediction. With just 2.5 years of data analysed, the orbital decay term for the Double Pulsar is not measured as precisely as that for PSR B1913+16. However, since the phase offset grows quadratically (Figure 4) and the effect of receiver noise decreases as $T^{1 / 2}$ where $T$ is the data span (assuming approximately uniform sampling), the precision of the $\dot{P}_{b}$ measurement should improve as $T^{5 / 2}$. Furthermore, J0737-3039A/B is much closer to the Sun than PSR B1913+16, thus reducing the magnitude and uncertainty of the correction due to differential Galactic acceleration. Also, using very long-baseline interferometry (VLBI), Deller et al. ${ }^{38}$ have shown that the transverse space velocity of the binary system is small, just $24_{-6}^{+9} \mathrm{~km} \mathrm{~s}^{-1}$, and so the Shklovskii correction to the observed $\dot{P}_{b}$ is also small and accurately known. For these reasons, the orbit-decay test with the Double Pulsar will not be limited by these corrections, at least for another decade or so. Beyond that, improved models for the Galactic gravitational potential can be expected from analysis of GAIA data ${ }^{108}$ and improved parallax and proper motion measurements may come from further VLBI observations, both reducing the uncertainty in these kinematic corrections.

Several other post-Keplerian parameters can in principle be observed in binary 


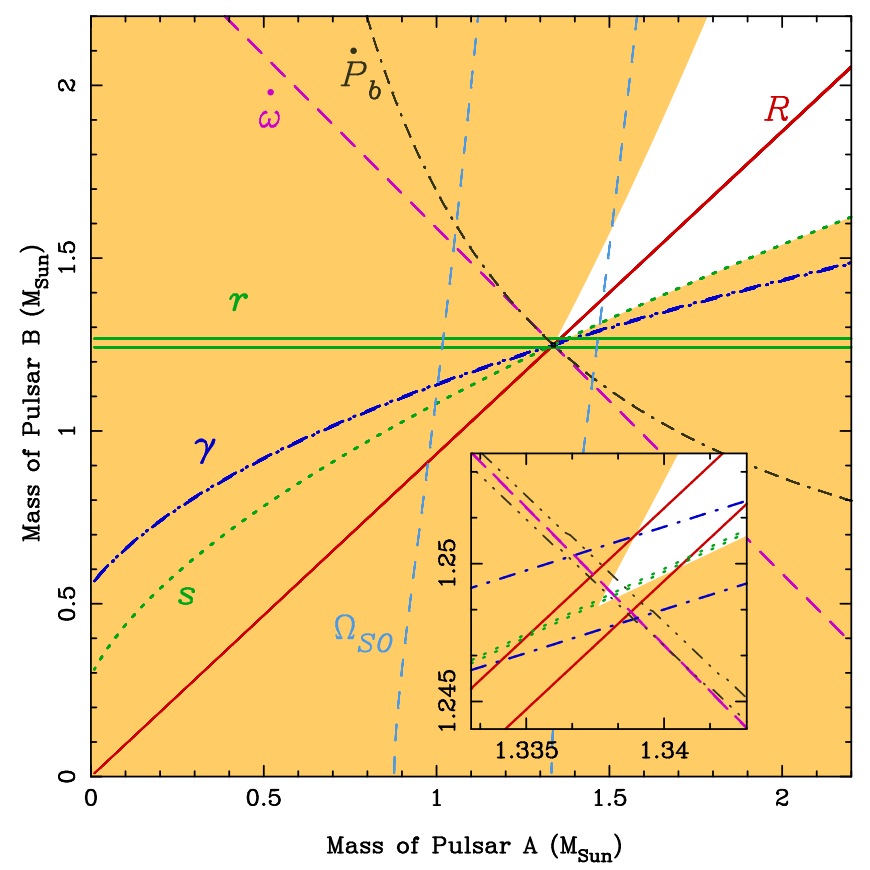

Fig. 8. Plot of companion mass $\left(m_{2}\right)$ versus pulsar mass $\left(m_{1}\right)$ for PSR J0737-3039 with observed constraints interpreted in the framework of GR. The inset shows the central region at an expanded scale, illustrating that GR is consistent with all constraints. (M. Kramer, private communication)

systems, $\stackrel{35}{, 35}$ testing other aspects of relativity. Kramer and Wex ${ }^{75}$ show that only one of these, $\delta_{\theta}$, which quantifies relativistic deformations of the binary orbit, is potentially measurable with the Double Pulsar system. Even this will take more than a decade to reach an interesting level of precision given reasonable expectations about future observations.

A potentially exciting prospect is to use measurement of higher-order terms for relativistic periastron precession to put constraints on the moment of inertia of a neutron star ${ }^{32}$ At the second post-Newtonian level, the periastron precession has three components:

$$
\dot{\omega}=\dot{\omega}_{1 p N}+\dot{\omega}_{2 p N}+\dot{\omega}_{S O}
$$

where $\dot{\omega}_{1 p N}$ is given by Equation $3 \dot{\omega}_{2 p N}$ is the 2nd post-Newtonian $(v / c)^{4}$ contribution and $\dot{\omega}_{S O}$ gives the contribution from spin-orbit coupling. ${ }^{16[32}$ This latter term depends on the moment of inertia and spin of the A pulsar (the spin angular momentum of the B pulsar is negligible) and can in principle be measured if the two leading components in Equation 9 can be measured to sufficient precision. Kramer and Wex ${ }^{75}$ show that, given expected advances in precision timing and astrometry, a significant result could be obtained in 20 years or so.

Spin-orbit coupling can also lead to a nonlinear variation in $\omega$ as a function of time and a time variation in the projected semi-major axis of pulsar $\mathrm{A}, a_{1} \sin i \frac{75}{7^{75}}$ 
However, the nonlinear terms depend on $\sin \theta$, where $\theta$ is the misalignment angle. As mentioned above, it seems as though $\theta_{A}$ is very small, so unfortunately these terms may be difficult to detect.

\subsubsection{Measured post-Keplerian Parameters}

Table 1 summarises the measured post-Keplerian parameters for the eleven pulsar binary systems where three or more post-Keplerian parameters have been measured. Since only two measurements are required to determine the stellar masses, these systems provide the opportunity for tests of theories of relativistic gravity. Of the eleven systems, in six cases the companion star is believed to be a neutron star, in a further four cases a white dwarf companion is more likely and in one case the companion is probably a main sequence star. This latter system, PSR J1903+0327, evidently has had an unusual evolutionary history as a triple system in which one component was ejected. ${ }^{[5]}$ It is currently not clear if there is a significant contribution to the observed $\dot{\omega}$ from kinematic effects and/or spin-orbit coupling, so the utility of this system for tests of relativistic gravity is limited. There a further ten pulsar systems in the literature where just two post-Keplerian parameters have been measured, enabling estimates of the stellar masses, but no tests of relativistic gravity.

Although an independent measurement of relativistic spin precession has been possible for just two systems so far, as shown in Table1, secular changes in observed pulse profiles have been observed for four other pulsars and attributed to geodetic precession of the pulsar spin axis.

\subsection{Tests of Equivalence Principles and Alternative Theories of Gravitation}

Pulsars and especially binary pulsars have unique advantages in testing theories of relativistic gravitation as a result of their often rapid spin, short orbital periods and the ultra-high density of the under-lying neutron stars. As we have shown above, GR has been amazingly successful in describing all measurements to date. Never-theless, investigations of quantum gravity and cosmology suggest that, in some regimes, extensions or modifications of GR may be required. This strongly motivates a search for departures from GR within existing experimental capabilities.

Gravitational theories have Equivalence Principles at their heart. The Weak Equivalence Principle (WEP) is basic to Newtonian gravity, stating that acceleration in a gravitational field is independent of mass or composition. The Strong Equivalence Principle (SEP) adds Lorentz invariance (no preferred reference frame) and position invariance (no preferred location) for both gravitational and nongravitational interactions. GR satisfies the SEP whereas other theories may violate the SEP or even the WEP in one or more respects.

Comparison of different gravitational theories has been greatly facilitated by the "parametrized post-Newtonian" (PPN) formalism which describes observable 
Table 1. Binary pulsars with three or more significant measured post-Keplerian parameters

\begin{tabular}{|c|c|c|c|c|c|c|}
\hline Pulsar/Parameter & J0437-4715 & J0737-3039A/B & J0751+1807 & J1141-6545 & $\mathrm{B} 1534+12$ & $\mathrm{~J} 1756-2251$ \\
\hline Peri. advance $\dot{\omega}\left({ }^{\circ} \mathrm{yr}^{-1}\right)$ & $0.016(8)$ & $16.8995(7)$ & - & $5.3096(4)$ & $1.7557950(19)$ & $2.58240(4)$ \\
\hline Time dilation $\gamma(\mathrm{ms})$ & - & $0.3856(26)$ & - & $0.773(11)$ & $2.0708(5)$ & $0.001148(9)$ \\
\hline Orb.P deriv. $\dot{P}_{b}\left(10^{-12}\right)$ & $3.73(6)^{\mathrm{b}}$ & $-1.252(17)$ & $-0.031(5)$ & $-0.403(25)$ & $-0.1366(5)$ & $-0.229(5)$ \\
\hline$s \equiv \sin i$ & $0.6746(28)^{\mathrm{b}}$ & $0.99974(39)$ & $0.90(5)$ & - & $0.9772(16)$ & $0.93(4)$ \\
\hline Comp. mass $m_{2}\left(M_{\odot}\right)$ & $0.254(18)$ & $1.2489(7)$ & $0.191(15)$ & - & $1.35(5)$ & $1.6(6)$ \\
\hline Geod. prec. $\Omega_{p}\left({ }^{\circ} \mathrm{yr}^{-1}\right)$ & - & $4.77(66)^{\mathrm{c}}$ & - & Note d & $0.59(10)$ & - \\
\hline Binary companion $^{\mathrm{a}}$ & He WD & NS & He WD & CO WD & NS & NS \\
\hline References & 141 & 74,20 & 96, 97 & 15 & 49 & 46 \\
\hline Pulsar/Parameter & J1807-2459B & $\mathrm{J} 1903+0327$ & $\mathrm{~J} 1906+0746$ & $\mathrm{~B} 1913+16$ & $\mathrm{~B} 2127+11 \mathrm{C}$ & \\
\hline Peri. advance $\dot{\omega}\left({ }^{\circ} \mathrm{yr}^{-1}\right)$ & $0.018339(4)$ & $0.0002400(2)^{b}$ & $7.5841(5)$ & $4.226598(5)$ & $4.4644(1)$ & \\
\hline Time dilation $\gamma(\mathrm{ms})$ & $26(14)$ & - & $0.470(5)$ & $4.2992(8)$ & $4.78(4)$ & \\
\hline Orb.P deriv. $\dot{P}_{b}\left(10^{-12}\right)$ & - & - & $-0.56(3)$ & $-2.423(1)^{\mathrm{b}}$ & $-3.96(5)$ & \\
\hline$s \equiv \sin i$ & $0.99715(20)$ & $0.9759(16)$ & - & - & - & \\
\hline Comp. mass $m_{2}\left(M_{\odot}\right)$ & $1.02(17)$ & $1.03(3)$ & - & - & - & \\
\hline Geod. prec. $\Omega_{p}\left({ }^{\circ} \mathrm{yr}^{-1}\right)$ & - & - & Note d & Note d & Note d & \\
\hline Binary companion ${ }^{\mathrm{a}}$ & $\mathrm{CO} \mathrm{WD}(?)$ & MS & NS(?) & NS & NS & \\
\hline References & 82 & 50 & 13941 & 145,143 & 62 & \\
\hline
\end{tabular}

Note: a: Binary companion types: CO WD: Carbon-Oxygen White Dwarf; He WD: Helium White Dwarf; MS: Main-sequence star; NS: Neutron star

b: Dominated or significantly biased by kinematic effects

c: For PSR J0737-3039B

d: Effects of precession observed, but no independent determination of $\dot{\Omega}_{p}$

or potentially observable phenomena in a theory-independent way. This formalism was first developed by Will and Nordtvedt ${ }^{150}$ for "weak-field" situations, that is, where $\epsilon \sim G M / R c^{2} \ll 1$, where $G$ is the Newtonian gravitational constant, $M$ and $R$ characterise the size and mass of the object and $c$ is the velocity of light. Many gravitational tests are performed within the solar system where $\epsilon \lesssim 10^{-5}$, firmly in the weak-field regime. However in the vicinity of a neutron star $\epsilon \sim 0.2$ and so strong-field effects are potentially important. A number of authors have considered the generalisation of the PPN formalism to strong-field situations (see, e.g., Refs 31, 148, 35) allowing investigation of these effects.

Some Lorentz-violating theories predict a dependence of the velocity of light on photon energy or polarisation! ${ }^{149}$ Pulsar observations can be used to limit these theories, but potentially stronger limits come from observations of gamma-ray bursts, polarised extra-galactic radio sources and the cosmic microwave background 95

A recent comprehensive review of observational limits on theories of gravitation by C. M. Will can be found in Ref. 149, Pulsar tests of relativistic gravitation have been reviewed by I. H. Stairs $\frac{129}{129}$ and, more recently, by N. Wex ${ }^{146}$ Further details on many of the topics discussed here may be found in these reviews. 


\subsubsection{Limits on PPN parameters}

The standard PPN formalism has ten parameters: $\gamma_{\mathrm{PPN}}, \beta_{\mathrm{PPN}}, \xi, \alpha_{1}, \alpha_{2}, \alpha_{3}, \zeta_{1}$, $\zeta_{2}, \zeta_{3}$ and $\zeta_{4}$. In GR $\gamma_{\mathrm{PPN}}$, describing space curvature per unit mass, and $\beta_{\mathrm{PPN}}$, describing superposition of gravitational fields, are unity and all others are zero. $\xi$ describes preferred location effects, the $\alpha_{n}$ preferred frame effects and the others describe violations of momentum conservation. ( $\alpha_{3}$ also may be non-zero in this case.) Pulsar observations do not directly constrain $\gamma_{\mathrm{PPN}}$ or $\beta_{\mathrm{PPN}}$ but are important in constraining many of the remaining PPN parameters and in fact currently place the strongest constraints on several parameters.

Damour and Schäfer ${ }^{33}$ recognised that wide-orbit low-eccentricity binary pulsars, which generally have a white-dwarf companion, could provide a valuable test of the SEP through a strong-field extension of the solar-system tests pioneered by Nordtvedt ${ }^{98}$ A violation of the SEP would cause bodies with different gravitational self-energy to fall at different rates in an external gravitational field. In a pulsar white-dwarf binary system, this results in a forced eccentricity in the direction of the gravitational field, that of the Galaxy in this case. This eccentricity is given by

$$
\mathbf{e}_{\mathrm{F}}=\frac{3}{2} \frac{\Delta \mathbf{g}_{\perp}}{\dot{\omega} a\left(2 \pi / P_{b}\right)}
$$

where $\mathbf{g}_{\perp}$ is the projection of the Galactic gravitational field on to the orbital plane, $\dot{\omega}$ is the relativistic periastron advance, $a$ is the orbit semi-major axis and $P_{b}$ the orbital period. The ratio of the gravitational mass $m_{g}$ and the inertial mass $m$ (which is exactly one if the SEP is obeyed) for body $i$ is described by

$$
\left(\frac{m_{g}}{m}\right)_{i}=1+\Delta_{i}=1+\eta_{N}\left(E_{g} / m c^{2}\right)_{i}+\eta_{N}^{\prime}\left(E_{g} / m c^{2}\right)_{i}^{2}+\ldots
$$

where $\eta_{N}$ is the Nordtvedt parameter, a function of several PPN parameters (see, e.g., Ref. (149) and $\Delta=\Delta_{1}-\Delta_{2}$. Violations of the SEP will result in non-zero $\Delta$.

Since Damour and Schäfer ${ }^{33}$ first proposed this method of testing SEP violations, the number of suitable pulsar - white-dwarf binary systems has increased greatly. Gonzalez et al ${ }^{53}$ combined data for 27 systems to place a $95 \%$ confidence upper limit on $|\Delta|$ of $4.6 \times 10^{-3}$ Since $E_{g} / m c^{2} \sim 0.1$ for a neutron star, this limit is not as strong as the weak-field limit on $\eta_{N} \lesssim 3 \times 10^{-4}$ from lunar-laser ranging! 151 However, it does enter the strong-field regime and test possible violations of the SEP that solar-system tests cannot reach.

The recent discovery by Ransom et al 104 of a remarkable triple system containing a pulsar, PSR J0337+1715, and two white-dwarf stars in essentially coplanar orbits, one in a relatively tight 1.6-day orbit with the pulsar and the other in a much wider 327-day orbit around the inner system, opens up the possibility of a much more sensitive test of the SEP. Precise timing observations of the pulsar have already shown that the motion of the inner system is strongly affected by the gravitational field of the outer white dwarf. The gravitational field of this star, which

${ }^{\mathrm{f}}$ This limit may be slightly under-estimated - see Wex!146 
has a mass of about $0.41 \mathrm{M}_{\odot}$, at the inner system is at least six orders of magnitude larger than the Galactic gravitational field at the position of a typical pulsar and so the effect of SEP violations may be expected to be correspondingly larger $\frac{51}{51}$ Observations over several orbital periods of the outer star will almost certainly be necessary to isolate any SEP-related effects from other orbit perturbations.

The wide-orbit low-eccentricity binary pulsars can also be used test for violations of local Lorentz invariance (LLI) of the gravitational interaction and momentum conservation that are described by the parameter $\alpha_{3}$ and its strong-field generalisation $\hat{\alpha}_{3}$. Bell and Damour ${ }^{13}$ show that such violations produce a forced eccentricity analogous to that produced by the Nordtvedt effect given by

$$
\left|\mathbf{e}_{F}\right|=\hat{\alpha}_{3} \frac{c_{p}|\mathbf{w}|}{24 \pi} \frac{P_{b}^{2}}{P} \frac{c^{2}}{G\left(m_{1}+m_{2}\right)} \sin \beta
$$

where $c_{p}$ is a dimensionless "compactness" parameter, for neutron stars about $0.2,31$ and $\beta$ is the angle between $\mathbf{w}$, the velocity of the system with respect to a reference frame defined (for example) by the cosmic microwave background, and the pulsar's spin axis. A similar analysis to that for the generalised Nordtvedt effect resulted in a $95 \%$ confidence limit of $\left|\hat{\alpha}_{3}\right|<4.0 \times 10^{-20}$, some 13 orders of magnitude lower than the best solar-system test ${ }^{[130}$ It is worth noting that the observed small scatter in the period derivatives of MSPs had already been used by Bell11 to limit $\left|\alpha_{3}\right|$ to $<5 \times 10^{-16}$.

Strong-field limits on the other two PPN parameters describing preferred frame effects, specifically LLI, $\hat{\alpha}_{1}$ and $\hat{\alpha}_{2}$, can also be obtained from low-eccentricity binary pulsar observations. ${ }^{[3]}$ Non-zero $\hat{\alpha}_{1}$ induces a forced eccentricity in the direction of motion of the binary system velocity $\mathbf{w}$, analogous to Nordtvedt $\hat{\alpha}_{3}$ tests discussed above, whereas non-zero $\hat{\alpha}_{2}$ induces a precession of the orbital angular momentum about $\mathbf{w}$. The best current limits come from observations of the binary pulsar systems PSRs J1012+5307 and J1738+0333, both of which have short orbital periods, $\sim 0.60$ and $\sim 0.35$ days respectively, and extremely low orbital eccentricities $e \lesssim 10^{-7 ! 121}$ Furthermore, both of these pulsars have optically identified binary companions which, together with proper motion measurements from the timing observations, allow the three-dimensional space velocity of the binary system to be determined. For these pulsars, the observational data span is sufficiently long ( $\gtrsim 10$ years) that relativistic periastron advance has significantly changed the orientation of the intrinsic eccentricity vector relative to the direction of any forced eccentricity, resulting in a potentially detectable change in the total eccentricity. The strongest limit on $\hat{\alpha}_{1}$ comes from observations of PSR J1738+0333 as shown in Figure 9, conservatively $\hat{\alpha}_{1}<4 \times 10^{-5}$. This is not only better than the best weak-field limit of $\alpha_{1}<2 \times 10^{-4}$ from lunar laser ranging ${ }^{93}$ but also constrains strong-field effects as well.

A precession of the orbital axis about the system velocity vector $\mathbf{w}$ induced by a non-zero value of $\hat{\alpha}_{2}$ is potentially observable as a time variation $\dot{x}$ in the projected semi-major axis of the pulsar orbit $x \equiv a_{1} \sin i$. $\dot{x}$ is one of the possible 

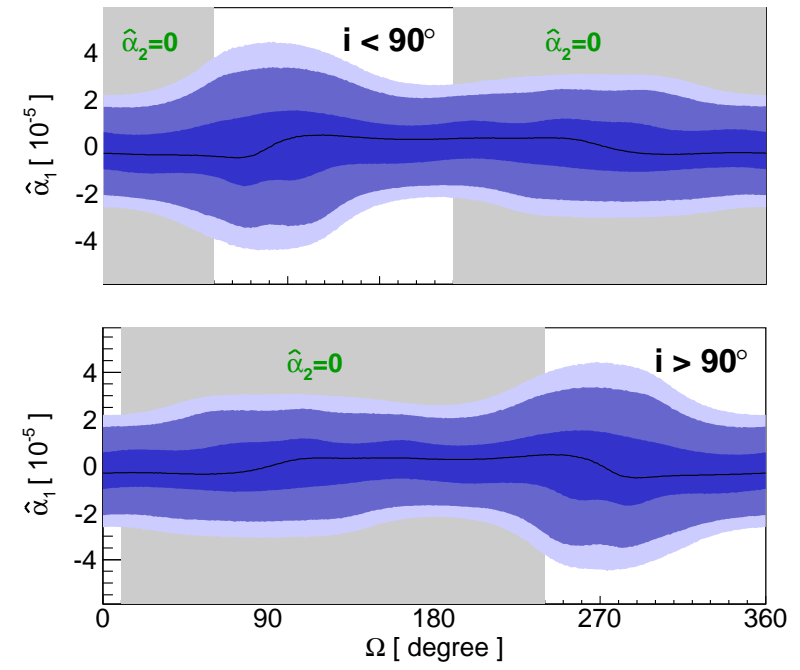

Fig. 9. Constraints on the strong-field PPN parameter $\hat{\alpha}_{1}$ from timing observations of the loweccentricity binary pulsar PSR J1738+0333. The constraints are a function of the unknown orientation of the binary system on the sky (described by the longitude of the ascending node $\Omega$ ) and the unknown sign of $\sin i$. If the PPN parameter $\hat{\alpha}_{2}$ is assumed to be zero, then only certain ranges of $\Omega$ are permitted. The shading corresponds to $68 \%, 95 \%$ and $99.7 \%$ confidence limits on $\hat{\alpha}_{1}$. (Ref. 121)

post-Keplerian parameters in standard timing solutions and significant values have been measured for both PSRs J1012+5307 and J1738+0333 ${ }^{121}$ There are several possible contributions to the observed $\dot{x}$ but in Ref. 121 it is shown that all of these are negligible in these systems except that due to the changing orbit inclination $i$ resulting from proper motion of the system. This is a function of the unknown $\Omega$ values and for certain $\Omega$ values there is no constraint. Consequently only a probabilistic limit on $\hat{\alpha}_{2}$ can be obtained. By combining results for the two pulsars, a $95 \%$ confidence limit of $\left|\hat{\alpha}_{2}\right|<1.8 \times 10^{-4}$ is obtained.

This is not as constraining as a solar-system limit $\left|\alpha_{2}\right|<2.4 \times 10^{-7}$ obtained from the present deviation of the Sun's spin axis from the solar-system orbit normal, ${ }^{99}$ a limit that rests on the assumption that the two axes were aligned at the time of formation of the solar system.

However, pulsars provide an even stronger constraint based on the stability of the spin axis of isolated pulsars. Any precession of the spin axis of a pulsar is likely to result in changes in the observed pulse profile (as observed with geodetic precession in binary pulsars as discussed in $\$ 2$ above). Shao et al ${ }^{120}$ compared mean pulse profiles for the isolated MSPs B1937+21 and J1744-1134 taken 10 - 12 years apart with the same observing system and found no perceptible change in the pulse width at $50 \%$ of the peak amplitude. They interpreted these results by assuming a circular beam profile for the main pulse in PSR B1937+21 and for PSR J1744-1134. All of 
the angles in the problem can be estimated from modelling of radio and gammaray observations of the pulsar, taken together with known direction of the system velocity $\mathbf{w}$ with respect to the cosmic microwave background, except the angle of the projected spin axis on the sky. Probability histograms for $\hat{\alpha}_{2}$ allowing for this unknown angle are shown in Figure10. The final result for the $95 \%$ confidence upper limit is $\left|\hat{\alpha}_{2}\right|<1.6 \times 10^{-9}$, about four orders of magnitude better than the limit described above based on orbital precession in pulsar binary systems and two orders of magnitude better than the limit based on solar spin precession. The assumption of circular beams in these MSPs is problematic, since there is good evidence for caustic enhancement in MSP pulse profiles, both radio and gamma-ray, ${ }^{105 / 54}$ which would tend to elongate the beam in the latitude direction, reducing the effect of precession on the observed pulse profiles. However, taking this into account would probably increase the limit by a factor less than ten, and so this limit would remain the best available.

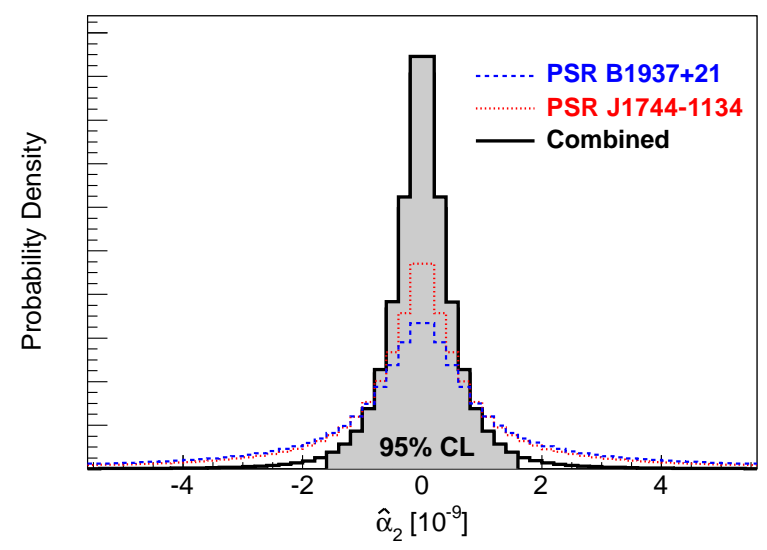

Fig. 10. Constraints on the strong-field PPN parameter $\hat{\alpha}_{2}$ based on the long-term stability of the mean pulse profiles for the isolated MSPs B1937+21 and J1744-1134. (Ref. 120)

The stable pulse profiles of PSRs B1937+21 and J1744-1134 have also been used to limit the PPN parameter $\xi$ describing local position invariance (LPI), also known as the Whitehead parameter, and its strong-field counterpart $\hat{\xi}$ (Ref. 122). The centripetal acceleration of Galactic rotation results in an anisotropy in the local gravitational field at a pulsar resulting in a precession of the pulsar spin vector around the direction of the Galactic acceleration with period.

$$
\Omega_{p}=\xi\left(\frac{2 \pi}{P}\right)\left(\frac{v_{G}}{c}\right)^{2} \cos \psi
$$

where $v_{G}$ is the velocity of Galactic rotation at the pulsar and $\psi$ is the (unknown) angle between the pulsar spin vector and the Galactic acceleration. Combining the results for the two pulsars in a way analogous to that for the $\alpha_{2}$ test described 
above, Shao and Wex ${ }^{122}$ obtain a $95 \%$ confidence limit $|\hat{\xi}|<3.9 \times 10^{-9}$. Even with the qualification about beam shapes mentioned above, this limit is at least two orders of magnitude better than the next best limit obtained from considering of the evolution of the solar spin-misalignment angle over the lifetime of the solar system. 122

The PPN parameter $\zeta_{2}$ is one of a number of parameters that may be non-zero in gravitational theories that violate conservation of total momentum $\frac{149}{1}$ A nonzero $\zeta_{2}$ would result in an acceleration of the centre of mass of a binary system that changes direction with periastron precession. This is best measured by looking for a change in the rate of orbital decay in a binary system with large periastron precession and a long data span. Will 147 used observations of PSR B1913+16 with a 15 -year data span to obtain a limit $\left|\zeta_{2}\right|<4 \times 10^{-5}$. Obviously, this test could be made more stringent using the long data spans now available for PSR B1913+16 and other double-neutron-star systems with high $\dot{\omega}$.

\subsubsection{Dipolar gravitational waves and the constancy of $G$}

As described above, because of its tiny eccentricity, the pulsar - white-dwarf binary system PSR J1738+0333 has played a significant role in limiting the PPN parameters describing preferred frame effects. This system is composed of two very different stars, the neutron star we see as a pulsar and the companion which we know to be a white dwarf of mass $0.181 \mathrm{M}_{\odot}$ through its optical identification. ${ }^{7}$ This and its short orbital period $(\sim 8.5 \mathrm{~h})$ make it an ideal system for testing theories of gravity that predict a dipolar component to gravitational-wave damping as well as general scalar-tensor theories. $\frac{52}{5}$

From analysis of about 10 years of timing data obtained at Parkes and Arecibo observatories, Freire et al ${ }^{52}$ find an observed rate of orbital decay for PSR J1738 +0333 of $\dot{P}_{b}=(-17.0 \pm 3.1) \times 10^{-15}$. To obtain the intrinsic rate of orbital decay, kinematic contributions from the differential acceleration of the binary system and the solar system in the Galactic gravitational field and the Shklovskii effect due to transverse motion must be subtracted, giving $\dot{P}_{b}^{\text {Int }}=(-25.9 \pm 3.2) \times 10^{-15}$. Since the orbital parameters and the masses of the two stars are well known, the orbit decay due to GR can be accurately determined: $\dot{P}_{b}^{\mathrm{GR}}=\left(-27.7_{-1.9}^{+1.5}\right) \times 10^{-15}$, leaving a residual orbit decay of $\dot{P}_{b}^{\text {Res }}=\left(2.0_{-3.6}^{+3.7}\right) \times 10^{-15}$.

This residual orbit decay is consistent with zero, which can be interpreted as a further confirmation of the accuracy of GR. However, because of the very different nature of the two stars in this binary system, this result also places strong constraints on theories of gravity that predict a dipolar component to gravitationalwave emission. Besides a dipolar component $\dot{P}_{b}^{\mathrm{D}}$, there are several other possible contributions to $\dot{P}_{b}^{\text {Int }}$ :

$$
\dot{P}_{b}^{\mathrm{Int}}=\dot{P}_{b}^{\dot{M}}+\dot{P}_{b}^{\mathrm{T}}+\dot{P}_{b}^{\mathrm{D}}+\dot{P}_{b}^{\dot{G}}
$$

where $\dot{P}_{b}^{\dot{M}}$ is due to mass loss from the binary system, $\dot{P}_{b}^{\mathrm{T}}$ is a term resulting from 
tidal effects on the white dwarf (tidal effects on the neutron star are negligible) and $\dot{P}_{b}^{\dot{G}}$ is decay resulting from a possible variation in the gravitational 'constant' $G$. ${ }^{34}$ Freire et al ${ }^{52}$ show that the likely $\dot{M}$ and tidal terms are small for this system, $\lesssim 10^{-15}$, and so the limit on $\dot{P}_{b}^{\text {Int }}$ is effectively a limit on $\dot{P}_{b}^{\mathrm{D}}+\dot{P}_{b}^{\dot{G}}$.

Within certain restrictions on strong-field effects, $\frac{52}{5}$ the dipole term is given by:

$$
\dot{P}_{b}^{\mathrm{D}}=-\frac{4 \pi^{2}}{P_{b}} T_{\odot} m_{c} \frac{q}{q+1} \kappa_{D} \mathcal{S}^{2}+\mathcal{O}\left(s^{3}\right)
$$

where $q=m_{1} / m_{2}$ is the mass ratio (with subscript 1 refering to the neutron star), $\mathcal{S}=s_{1}-s_{2}$ is the difference in "sensitivity" $s$ of the mass of each body to a scalar field $\phi$, where

$$
s \equiv\left(\frac{d \ln m(\phi)}{d \ln \phi}\right)
$$

and $\kappa_{D}$ is a body-independent constant that describes the dipole self-gravity contribution in a given theory of gravity (see, e.g., Ref. 149). The sensitivity $s_{i}$ depends on the stellar equation of state and, for neutron stars, is typically about 0.15 , whereas for a white dwarf it is $\sim 10^{-4}$. Therefore if $\kappa_{D}$ is non-zero, dipole radiation will contribute to the orbit decay.

The remaining term in the residual orbit decay is that due to possible variations in $G$. In weak gravity, $\dot{G} / G$ has been constrained to be less than $4 \times 10^{-13} \mathrm{yr}^{-1}$ from lunar laser ranging experiments, $\frac{60}{6}$ giving

$$
\dot{P}_{b}^{\dot{G}}=-2 \frac{\dot{G}}{G} P_{b}<0.8 \times 10^{-15} .
$$

and hence $\left|\kappa_{D}\right|<2 \times 10^{-4}$. However, it is also possible to obtain independent estimates of the effects of dipole radiation and $\dot{G}$ by combining the J1738+0333 results with those for PSR J0437-4715 $\stackrel{39}{[}$ This southern pulsar is in a wider orbit than PSR J1738+0333 and hence has a different mix of the dipole and $\dot{G}$ components, allowing them to be separated. After accounting for the fact that a changing $G$ will also change the stellar masses, $\frac{100}{10}$ the formal results are $\dot{G} / G=(-0.6 \pm 1.6) \times 10^{-12} \mathrm{yr}^{-1}$ and $\kappa_{D}=(-0.3 \pm 2.0) \times 10^{-4}$, both effectively upper limits. While the limit on $\kappa_{D}$ is the best available, the derived limit on $\dot{G} / G$ is about an order of magnitude weaker than the result (actually a limit on the variation of $G M_{\odot}$ ) from the Mars Reconnaissance Orbiter ${ }^{71}$ and from lunar laser ranging! 60

Interestingly, pulsars have provided two other independent limits on $\dot{G} / G$. Thorsett ${ }^{136}$ used determinations of neutron star masses from timing observations of double-neutron-star systems that formed many gigayears ago. In standard formation scenarios, the mass of a neutron star depends on the Chandrasekhar mass, the maximum possible mass of a white dwarf star, just prior to the collapse to a neutron star. The Chandrasekhar mass is proportional to $G^{-3 / 2}$ and so the observed small range of neutron star masses implies that $\dot{G} / G<4 \times 10^{-12}$. This limit has been somewhat weakened by recent discoveries of both less massive and more massive neutron stars in pulsar binary systems (see Ref. 70 for a recent review). 
The very small observed rate of change of pulsar period $\dot{P}$ observed in some pulsars (after correction for kinematic effects) may be used to set a further independent limit ${ }^{153}$ A variation in $G$ will result in an inverse variation in the stellar moment of inertia, with the exact relation depending on the neutron-star structure. If the observed (intrinsic) $\dot{P}$ is entirely attributed to this effect, a limit of $\dot{G} / G \lesssim 2 \times 10^{-11}$ is obtained.

\subsubsection{General scalar-tensor and scalar-vector-tensor theories}

Many alternate theories of gravity can be expressed in a "tensor-scalar" framework in which a scalar field $\phi$ contributes to the "physical metric" $\tilde{g}_{\mu \nu}$ through a coupling function $A(\phi)$ :

$$
\tilde{g}_{\mu \nu} \equiv A^{2}(\phi) g_{\mu \nu}
$$

where $g_{\mu \nu}$ is the usual tensor metric. The coupling constant may be expressed in different ways $\frac{149}{149}$ one of which is as an expansion around the asymptotic value of the scalar field $\phi_{0}$ :

$$
\ln A(\phi)=\ln A\left(\phi_{0}\right)+\alpha_{0}\left(\phi-\phi_{0}\right)+\beta_{0}\left(\phi-\phi_{0}\right)^{2}+\ldots
$$

(Ref. 31). For GR, $\alpha_{0}=\beta_{0}=0$. In the well-known example of a tensor-scalar theory, the Brans-Dicke theory, the scalar coupling is described by a single parameter $\omega_{B D}$. For $\omega_{B D} \rightarrow \infty$, the Brans-Dicke theory approaches GR. For this theory $\alpha_{0}=$ $1 /\left(2 \omega_{B D}+3\right)$ and $\beta_{0}=0$. In other theories both the linear and quadratic terms in Equation 19 (and higher-order terms) may be non-zero.

The various observational constraints on PPN and post-Keplerian parameters can be expressed as limits in the $\left(\alpha_{0}, \beta_{0}\right)$ space for tensor-scalar theories as shown in Figure $111^{52}$ The Cassini experiment ${ }^{14}$ placed a strong limit on the PPN parameter $\gamma_{P P N}=1+(2.1 \pm 2.3) \times 10^{-5}$ which translates to a limit on $\left|\alpha_{0}\right|$ of about 0.003 . Only the limits on dipole gravitational radiation from the asymmetric binary systems PSR J1141-6545 $5^{15}$ and PSR J1738+0333 $3^{\sqrt{52}}$ rival the Cassini limit over most of the space. Since the precision of these measurements will increase with time, it seems likely that ultimately binary systems such as these will provide the strongest constraints on tensor-scalar theories.

PSR J0348+0432 and its white-dwarf companion form another asymmetric binary system, one that is distinguished by its very short orbital period ( 2.46 hours) and massive neutron star $\left(2.01 \pm 0.04 \mathrm{M}_{\odot}\right){ }^{[6}$ The high neutron-star mass is of interest since some scalar-tensor theories predict a strongly non-linear relationship between the strength of dipole GW emission and neutron-star mass or self-gravity (cf., Equation 15). For most of the parameter space of the class of theories discussed by Freire et al., 52 the limits on effective scalar coupling from PSR J0348+0432 are currently not as strong as those from PSR J1738+0333 but, because of the high neutron-star mass, they place stronger limits on some other theories with a greater degree of non-linear coupling $\frac{146}{6}$ 


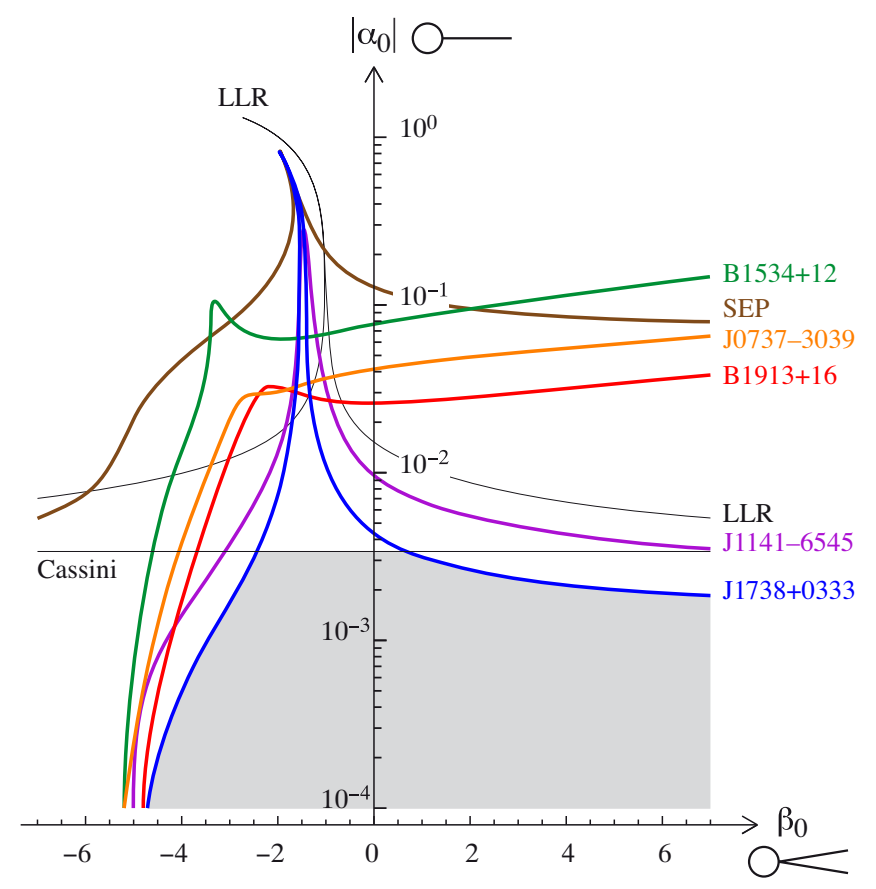

Fig. 11. Constraints on the scalar-field parameters in the $\left(\left|\alpha_{0}\right|, \beta_{0}\right)$ plane from various observational tests. Only the region below each line is allowed by the corresponding test. "SEP" refers to the test of the strong equivalence principle based on low-eccentricity pulsar - white-dwarf binary systems, $\frac{53}{4 L L R " ~ r e f e r s ~ t o ~ l u n a r ~ l a s e r ~ r a n g i n g ~ r e s u l t s ~} 151$ and Cassini to the "Shapiro delay" experienced by signals to and from the Cassini spacecraft (on its way to Saturn) as its line of sight passed close to the Sun $\frac{14}{14}$ Other binary-system tests are labelled according to the pulsar concerned. In GR, both $\alpha_{0}$ and $\beta_{0}$ are zero. (Ref. 52)

Bekenstein 10 has proposed a relativistic generalisation of the so-called "MOND" theory of gravity ${ }^{91}$ that seeks to avoid the need for dark matter in galactic dynamics. The generalisation invokes an additional vector field and hence is known as a tensorvector-scalar theory. Such theories relax some of the constraints on tensor-scalar theories, in particular, the dipole radiation constraints, and allow significantly larger values of $\alpha_{0}$. However, as shown by Freire et al., $\frac{52}{5}$ the binary pulsar results still significantly constrain theories of this type and in fact are more constraining than solar-system tests. With future observations, binary pulsar tests have the potential to make this class of theories untenable.

In another example of the use of pulsar observations, especially the limits on dipolar-GW radiation, to place limits on gravitational theories, Yagi et al ${ }^{155}$ have strongly limited the allowed parameter space for the LLI-violating "Einstein-Æther" and the "Khronometric" theories. 


\subsection{Future Prospects}

As described above, GR has provided an accurate description of all pulsar timing results obtained so far. However, continued refinement of existing methods and development of new tests is highly desirable. Continued pulsar timing measurements, especially with the advent of new and more sensitive observing facilities such as the 500-m Arecibo-type FAST radio telescope in China ${ }^{94}$ and the Square Kilometre Array $(S K A)$ in South Africa and Australia ${ }^{24}$ will certainly improve on existing limits and enable new tests of gravitational theories. They may even demonstrate a failure of GR and hence a need for a modified or conceptually different theory of gravity. Conversely, if GR is assumed to be valid, results of astrophysical significance can be deduced from the observations. For example, as described in Section 2.1.3. observations of higher-order terms in the relativistic perturbations may enable a measurement of the moment of inertia of a neutron star.

These more sensitive radio telescopes can also be used to search for previously unknown pulsars and binary systems that are suitable for tests of gravitational theories. Past experience has shown that pulsar searches repeatedly turn up new classes of object. This potential is wonderfully illustrated by the recent discovery of the pulsar triple system PSR J0337+1715 which promises to provide a strong limit on violations of the Strong Equivalence Principle. A dream for such searches is the discovery of a pulsar in a close orbit around a black hole as this would offer much more stringent tests of gravity in the strong-field regime ${ }^{80}$ Discovery of a pulsar orbiting the black hole at the centre of our Galaxy with an orbital period of a few months or less could even allow a test of the so-called "no-hair" theorem for black holes 81

\section{The Quest for Gravitational-Wave Detection}

The direct detection of the gravitational waves (GWs) predicted by Einstein's general theory of relativity and other relativistic theories of gravity is one of the major goals of current astrophysics. As described in $\$ 2$ above, we have excellent evidence from the orbital decay of binary systems for the existence of GWs at the level predicted by GR, but up to now there has been no direct detection of the changing curvature of spacetime induced by a passing GW. This changing curvature induces a change in the proper distance between two test masses, described by the gravitational strain $h=\delta L / L$. The problem is that, for any likely source, $h$ is tiny. For example, the $L I G O$ gravitational-wave detector ${ }^{2]}$ hopes to detect the merger of two neutron stars at a distance of $100 \mathrm{Mpc}$ for which $h \sim 10^{-22}$, a change in the length of its $4-\mathrm{km}$ arms of $10^{-18} \mathrm{~m}$ or $10^{-3}$ of the diameter of a proton!

Pulsar timing can measure a change in the proper distance between the pulsar and the telescope. Systematic changes in timing residuals for a given pulsar reflect unmodelled changes in the effective time of emission, the pulsar position, the propagation path or the position of the telescope. With care, and with observations of multiple pulsars, residual delays due to changing proper distances can be 
isolated, effectively giving us a set of interferometers with baselines of $\gtrsim 10^{16} \mathrm{~km}$ ! However, even in the best cases, we can only measure the interferometer "phase" to about $100 \mathrm{~ns}$, so that the limiting strain is about $10^{-18}$. Unlike ground-based laser-interferometer systems, which are most sensitive to GW signals with frequencies around $100 \mathrm{~Hz}$, pulsar timing systems are most sensitive to signals with frequencies around the inverse of the data span, typically a few nanohertz. Potential sources of detectable GWs in this low-frequency band include super-massive blackhole $(\mathrm{SMBH})$ binary systems in distant galaxies and cosmic strings in the early Universe.

\subsection{Pulsar Timing Arrays}

The effect of GWs on pulsar timing signals was first considered by Sazhin 112 and Detweiler $\frac{42}{2}$ with the latter being the first to consider the effect of a GW from a distant source passing over a pulsar and the Earth. For this case, it can be shown that the net effect on the observed pulsar arrival times is simply the difference between the effect of the GW passing over the pulsar and the effect of the GW passing over the Earth. For a GW travelling in the $\hat{z}$ direction, the redshift $z$ of the pulse frequency $\nu$ for a pulsar at distance $d$ with direction cosines $(\alpha, \beta, \gamma)$ is given by:

$$
z(t)=\frac{\nu_{0}-\nu(t)}{\nu_{0}}=\frac{\alpha^{2}-\beta^{2}}{2(1+\gamma)} \Delta h_{+}+\frac{\alpha \beta}{1+\gamma} \Delta h_{\times}
$$

where $\Delta h_{A}=h_{A}^{p}-h_{A}^{E}$, with $A$ representing the two possible wave polarisation states $(+, \times)$ in GR, and $h_{A}^{p}(t-d / c)$ and $h_{A}^{E}(t)$ the gravitational strain at the pulsar and the Earth, respectively $g$ The observed timing residuals are then given by the integral of the redshift,

$$
R(t)=\int_{0}^{t} z\left(t^{\prime}\right) d t^{\prime}
$$

The coefficients multiplying the $\Delta h_{+}$and $\Delta h_{\times}$terms are the "antenna patterns" for the two polarisations as illustrated in Figure 12. A pulsar located in the GW propagation direction has zero sensitivity to the GW since its direction cosines $\alpha$ and $\beta$ are zero. Furthermore, despite the $(1+\gamma)$ term in the denominator of Equation 20 . the response for a pulsar exactly in the $-z$ direction (the same direction as the $\mathrm{GW}$ source) is also zero. This comes about because of a cancellation of the $(1+\gamma)$ term by the expansion of $\Delta h_{A}$ when $1+\gamma \ll 1$ (cf. Ref. 78).

A pulsar timing array (PTA) consists of a set of pulsars spread across the sky which have precise timing measurements over a long data span. The detection of GWs by PTAs depends on the correlated timing residuals for different pulsars given

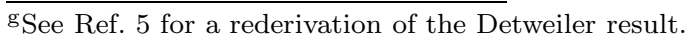


Fig. 12. Effective "antenna pattern" for detection of a GW with pulsar timing. The wave is propagating in the $+z$ direction and is assumed to have the + polarisation. The pattern for the $x$ polarisation is the same but rotated by $45^{\circ}$ about the $z$ axis. (Ref. 25)

by the Earth term $h_{A}^{E}(t)$ in Equation 20, GWs passing over the pulsars produce uncorrelated residuals because of both the retarded time and the different GW environment for each pulsar. Also the pulsars themselves have uncorrelated timing noise at some level, either intrinsic or resulting from uncorrected variations in interstellar delays. Because the expected GW strain is so weak, only MSPs have sufficient timing precision to make GW detection with PTAs feasible.

For an isolated source of continuous GWs, say an SMBH binary system in a nearby galaxy, in principle, both the Earth term and and the pulsar term in Equation 20 could be detected. For a rapidly evolving source, the pulsar term and the Earth term may be at different frequencies because of the retarded time of the pulsar term (see, e.g., Ref. 65). They can then be added incoherently to increase the detection sensitivity. For a non-evolving source, i.e., $\delta f_{b} \ll 1 / T$ where $\delta f_{b}$ is the change in binary orbital frequency over the pulsar timing data span $T$, in principle the pulsar term and the Earth term could be summed coherently for optimal sensitivity. As is discussed further in Section 3.4 below, unfortunately we currently don't know enough pulsar distances to sufficient accuracy to make this coherent addition possible. In a PTA, the pulsar terms therefore add with random phase, washing out the fringes in the antenna pattern (see also Ref. 78) and adding "self-noise" to the signal from the Earth term. Since the antenna pattern (Figure 12) has a maximum for pulsars roughly in the same direction as the GW source, the maximum response 
of a PTA is toward the greatest concentration of pulsars in the array.

A stochastic background of nanohertz GW from many SMBH binary systems in distant galaxies is likely to be the signal first detected by PTAs. To a first approximation, this background is also likely to be statistically isotropic, i.e., the expectation value $\left\langle h^{2}\right\rangle$ is independent of direction when averaged over typical data spans. Hellings and Down ${ }^{55}$ were the first to show that, in this case, the correlation between GW-induced timing residuals for two pulsars separated by an angle $\theta$ on the sky is dependent only on $\theta$ and not on the sky positions of the two pulsars. The zero-lag correlation function, commonly known as the Hellings \& Downs curve and obtained by integrating the product of the antenna patterns (Figure 12) for the two pulsars over all possible GW propagation directions, is given by:

$$
c_{\mathrm{HD}}=\frac{1}{2}+\frac{3 x}{2}\left(\ln x-\frac{1}{6}\right),
$$

where $x=(1-\cos \theta) / 2$, and is plotted in Figure 13, $c_{\mathrm{HD}}$ goes negative for angular separations around $90^{\circ}$ and then positive again for pulsars that are more-or-less opposite on the sky - this is a direct consequence of the quadrupolar nature of GWs. It is also important to note that the limiting value as $\theta \rightarrow 0$ is 0.5 , not 1.0. This is a consequence of the fact that the pulsar terms in Equation 20 are uncorrelated and, on average, of equal amplitude to the Earth term. The scatter in the simulated correlations results from the random phases of the pulsar terms and illustrates the "self-noise" that limits the sensitivity of PTA experiments in the strong-signal limit.

\subsection{Nanohertz Gravitational-Wave Sources}

\subsubsection{Massive black-hole binary systems}

There is good evidence that massive black holes form in the centre of galaxies at very early times (see, e.g., Ref. 140) and also that merger events play a major role in galaxy growth (see, e.g., Ref. 132). When two galaxies, each containing a central massive black hole, merge, dynamical friction will result in the two black holes migrating to the centre of the merged galaxy to form a binary system, with an estimated timescale for the migration of the order of giga-years (see, e.g., Ref. 68). When the binary separation is less than about $1 \mathrm{pc}$, loss of energy to GWs becomes the dominant orbital decay mechanism and the binary system will ultimately coalesce, becoming a strong GW source as it spirals in. There remains much controversy about the efficiency of orbital decay mechanisms as the binary separation approaches a parsec - known as the "last parsec problem". Some (see, e.g., Ref. 69, 107) argue that dissipation mechanisms will quickly move the binary system through this phase, whereas others (see, e.g., Ref. 27) argue that the binary is likely to stall at separations where the gravitational decay is ineffective. Detection of a stochastic GW background (GWB) would resolve this issue.

Since large numbers of binary systems with different orbital periods contribute to 


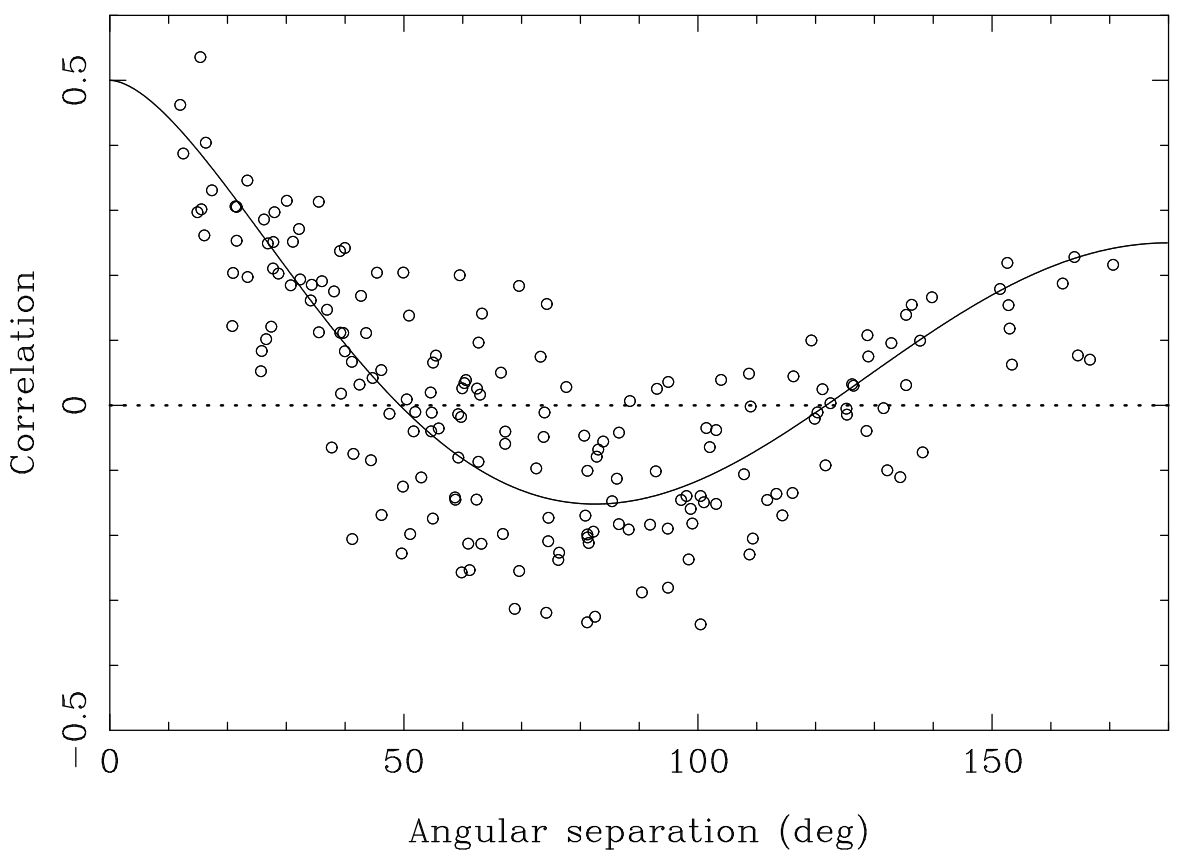

Fig. 13. The Hellings \& Downs correlation function, i.e., the correlation between timing residuals for pairs of pulsars as a function of their angular separation for an isotropic stochastic background of GWs. Also shown are simulated correlations between the 20 pulsars of the Parkes PTA for a single realisation of a strong GW signal that dominates all other noise contributions. (Ref. [58)

the GWB, it is a broadband signal which is best described in the spectral domain. It is convenient to express the amplitude of the GW signal in terms of the dimensionless "characteristic strain", defined by

$$
h_{c}=2 f|\tilde{h}(f)|
$$

where $\tilde{h}(f)$ is the Fourier transform of $h(t)$ and $f$ is the GW frequency. (Note that, for a circular binary system, the frequency of the emitted GW is twice the binary orbital frequency $f=2 f_{b}$.) Two other quantities that are often used to parameterise GW spectra and detector spectral sensitivities are the square root of the one-sided strain power spectral density

$$
S_{h}^{1 / 2}(f)=h_{c} f^{-1 / 2}
$$

and the GW energy density as a fraction of the closure energy density of the Universe

$$
\Omega_{G W}=\frac{2 \pi^{2}}{H_{0}^{2}} f^{2} h_{c}^{2}(f)
$$

where $H_{0}$ is the Hubble constant.

In order to understand the astrophysical implications of results obtained from PTA experiments, it is necessary to have estimates of the likely strength of signals from potential sources of nanohertz GW. For a cosmological population of SMBH 
binary systems at luminosity distance $D_{L}$ and redshift $z$, the local energy density in $\mathrm{GW}$ at frequency $f$ is given by:

$$
f S_{E}(f)=\int_{0}^{\infty} d z \int_{0}^{\infty} d M_{c} \frac{d^{2} n}{d z d M_{c}} \frac{1}{(1+z)} \frac{1}{D_{L}^{2}} \frac{d E_{g}}{d \ln f_{r}}
$$

where $M_{c}=\left(M_{1} M_{2}\right)^{3 / 5}\left(M_{1}+M_{2}\right)^{-1 / 5}$ is the binary chirp mass and $M_{1}$ and $M_{2}$ are the masses of the binary components, $d^{2} n /\left(d z d M_{c}\right)$ is the comoving density of binary systems with redshift and chirp mass between $z$ and $z+d z$ and $M_{c}$ and $M_{c}+d M_{c}$, respectively, and $d E_{g} / d \ln f_{r}$ is the total energy emitted by a single binary system in the logarithmic frequency interval $d \ln f_{r}$, where $\left.f_{r}=f(1+z)\right)^{103|115| 92}$ The local GW energy density is related to the local characteristic strain by

$$
f S_{E}(f)=\frac{\pi c^{2}}{4 G} f^{2} h_{c}^{2}(f)
$$

and for a circular binary system

$$
\frac{d E_{g}}{d \ln f_{r}}=\frac{G^{2 / 3} \pi^{2 / 3}}{3} M_{c}^{5 / 3} f_{r}^{2 / 3}
$$

Therefore we have

$$
h_{c}^{2}(f)=\frac{4 G^{5 / 3}}{3 \pi^{1 / 3} c^{2}} f^{-4 / 3} \int_{0}^{\infty} d z \int_{0}^{\infty} d M_{c} \frac{d^{2} n}{d z d M_{c}} \frac{1}{(1+z)^{1 / 3}} \frac{1}{D_{L}^{2}} M_{c}^{5 / 3} .
$$

As Phinney $\sqrt{103}$ has emphasised, the result that $h_{c} \propto f^{-2 / 3}$ for a cosmological population of circular binary systems decaying through GW emission is quite general and independent of any particular cosmology, black-hole mass function or galaxy merger scenario. Consequently the spectrum of the GWB is often parameterised as follows:

$$
h_{c}(f)=A_{1 \mathrm{yr}}\left(\frac{f}{f_{1 \mathrm{yr}}}\right)^{\alpha}
$$

where $f_{1 \mathrm{yr}}=(1 \mathrm{yr})^{-1}, A_{1 \mathrm{yr}}$ is the characteristic strain at $f_{1 \mathrm{yr}}$ and $\alpha=-2 / 3$ for the case described above. For pulsar timing experiments, the one-sided power spectrum of the timing residuals is given by

$$
P(f)=\frac{1}{12 \pi^{2}} \frac{1}{f^{3}} h_{c}^{2}(f) .
$$

Consequently, a GWB produces a very "red" modulation of the timing residuals with a spectral index of $-13 / 3$ for $\alpha=-2 / 3$.

In order to estimate the likely strength of this modulation, the factor $d^{2} n /\left(d z d M_{c}\right)$ in Equation 29 must be evaluated. This requires a prescription for the cosmological evolution of massive black-hole binary systems in galaxies. Different approaches to this problem have been taken by different authors. An early paper by Jaffe and Backer ${ }^{\sqrt{63}}$ used observational constraints on close galaxy pairs coupled with a black-hole mass function, whereas another early paper by Wyithe and Loeb ${ }^{154}$ used a prescription for merger of dark-matter halos coupled with different scenarios for growth of massive black holes in galaxies. The latter approach was 
developed further by Sesana et al ${ }^{115}$ who showed that the GWB spectrum steepens at frequencies above about $10^{-8} \mathrm{~Hz}$ since the number of binary systems contributing to the background at these frequencies becomes small. This is illustrated in the left panel of Figure 14 which shows that binary systems at $z \lesssim 2$ contribute most of the strain to the GWB. The right panel shows that massive binary systems with $M_{c} \gtrsim 10^{8} \mathrm{M}_{\odot}$ contribute most of the low-frequency GWB but that only systems with $M_{c} \lesssim 10^{8} \mathrm{M}_{\odot}$ contribute to the high-frequency end. Importantly, at the highmass end, only a few systems contribute to the GWB. As shown in Figure 15, this leads to a steepening and large uncertainties in the expected GWB spectrum at frequencies $\gtrsim 10^{-8} \mathrm{~Hz}$. These results were confirmed and extended by Sesana et al!116 and Ravi et al ${ }^{106}$ using the Millenium simulation of the cosmological evolution of dark matter structures ${ }^{128}$ to define a merger history together with various prescriptions for galaxy and black-hole formation and growth.
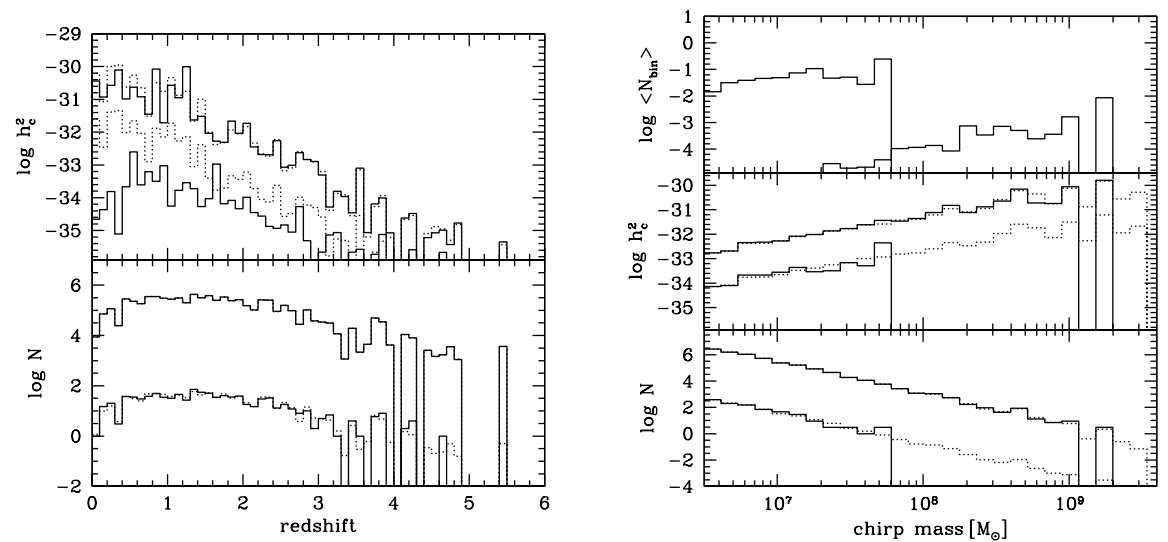

Fig. 14. Left panel: Number of binary black-hole systems and their contribution to the characteristic strain $h_{c}$ of the GWB as function of source redshift $z$. The solid lines are results from a Monte Carlo approach and the dotted lines are from a semi-analytic analysis. In both panels, the upper histograms are for a GW frequency $f=8 \times 10^{-9} \mathrm{~Hz}$ and the lower histograms for $f=10^{-7} \mathrm{~Hz}$. Right panel: The lower two panels are as for the left panel but as a function of source chirp mass $M_{c}$. The upper panel shows the number of frequency bins spanned by the chirp over the 5 -year span of the simulation. (Ref. 115)

While there is some consensus about the form of the GWB spectrum, there remain significant uncertainties. For example, Ravi et al ${ }^{107}$ consider the effects of the stellar environment on the late evolution of massive black-hole binary systems in the cores of galaxies and conclude that the effect of dynamical friction is important, both in extracting energy from the binary system and inducing an eccentricity. Both of these have the effect of reducing the strength of the predicted GWB, especially at the low-frequency end, consequently making its detection by PTAs more difficult. On the other hand, McWilliams et al. ${ }^{89}$ argue for a model in which all black- 
hole growth is by merger rather than by accretion after coalescence, which is the main contributor to black-hole growth in the models discussed above. This leads to predictions of a significantly larger GWB characteristic strain compared to previous predictions and, hence, the imminent detection of the GWB by PTAs.

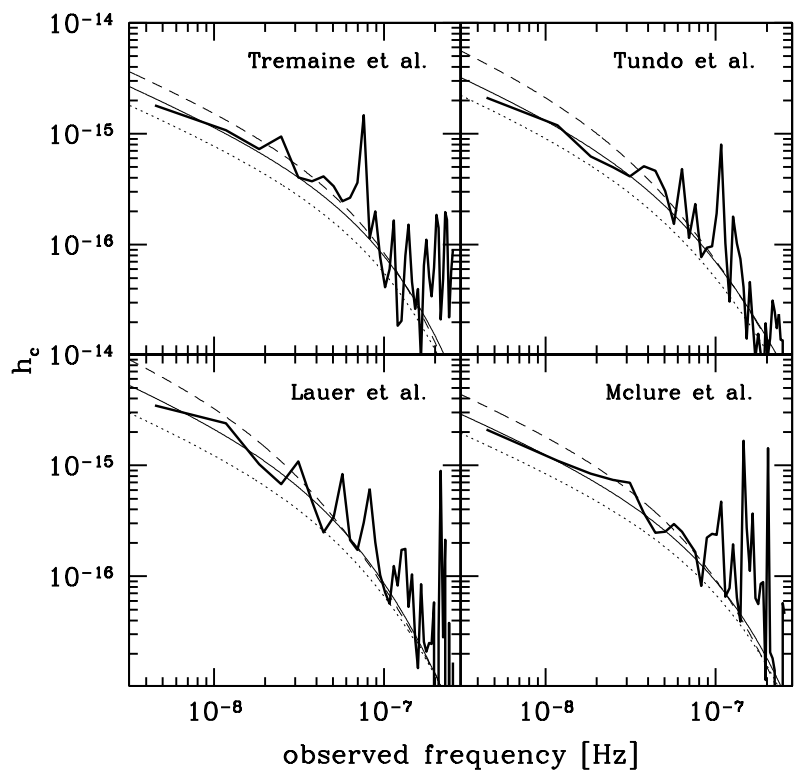

Fig. 15. Characteristic strain spectrum for the GWB based on different prescriptions for blackhole growth by accretion between mergers (solid, dashed and dotted lines) and different black-hole mass functions (different panels). (Ref. 116)

As Figure 15 indicates, there is a possibility that the nearby universe could contain a massive black-hole binary system with an orbital period of the order of a few years that actually dominates the nanohertz GW spectrum. This opens up the exciting prospect of the GW detection and study of an isolated supermassive black-hole binary system using pulsar timing and even the possibility of detection and study of the system in the electromagnetic bands - so-called "multi-messenger" astronomy. Searches for binary GW sources will be described in $\$ 3.3$ and the prospects for their detailed study will be discussed in $\$ 3.4$.

\subsubsection{Cosmic strings and the early Universe}

Cosmic strings and the related cosmic super-strings are one-dimensional topological defects which may have formed in phase transitions in the early Universe. Cosmic strings occur in standard field-theory inflation models, whereas superstrings are found in brane inflationary models. The idea that such strings will oscillate and 
hence emit GWs was first proposed by Vilenkin! ${ }^{142}$ Such oscillations may contribute to the stochastic GWB (see, e.g., Ref. 23) or generate bursts of GW radiation from string cusps and kinks (see, e.g., Ref. 36). The amplitude of GWs from cosmic strings is dependent on a large number of poorly known (or unknown) parameters and hence is very uncertain (see, e.g., Ref. 111). Key parameters are the string tension $\mu$, usually parameterised by the dimensionless quantity $G \mu / c^{2}$, and the size $\alpha$ of string loops relative to the horizon radius at the time of birth. Other significant parameters for the GWB are the intrinsic spectral index $q$ of the GW emission, a characteristic node number $n_{*}$ for the high-frequency cutoff in the emission spectrum and the probability $p$ of "intercommutation", that is, intersecting strings dividing and the two parts exchanging. Such intercommutation can, for example, form two smaller loops from an intersecting twist in a larger loop. For standard strings, $p=1$ but it may be less for superstrings.

Vibrating cosmic strings are likely to decay by emission of GWs in a series of harmonics with fundamental frequency $2 c / l$, where $l$ is the length of the loop, with an initial value $\alpha D_{H}$, where $D_{H}$ is the horizon distance at the time of loop creation. The rate of energy loss for vibration mode $n$ of a given loop is:

$$
\frac{d E_{G W}}{d t}=\Gamma \frac{n^{-q}}{\sum_{m=1}^{\infty} m^{-q}} G \mu^{2} c
$$

where $\Gamma$ is factor depending on the shape of the loop, typically about 50.111 As the loop loses energy, it shrinks and eventually disappears. The creation of loops through intercommutation and their decay through GW emission sets up an equilibrium distribution of loop sizes. Sanidas et al ${ }^{111}$ compute the number density of loops as a function of loop length and time and hence, using Equation 32, the predicted spectrum of the GWB from string loops as a function of the various parameters. As Figure 16 shows, the spectrum is very broad, extending all the way from nanohertz to Megahertz. Since the lowest $(n=1)$ frequency is proportional to the string length, the weaker and smaller loops do not contribute to the nanohertz background.

\subsubsection{Transient or Burst $G W$ sources}

The prime target of ground-based laser-interferometer GW detectors is the burst emitted at the coalescence of a double-neutron-star system. Such a burst is intense for just a few milliseconds and clearly cannot be detected by PTA experiments which typically are sensitive to signals of duration between a few weeks and a few years. Possible sources of longer-duration bursts are coalescence of SMBH binary systems, highly eccentric massive black-hole binary systems, and formation and decay of cusps and kinks in cosmic strings.

A major difference between detection of GW bursts and continuous GW sources is that there is generally no interference between the Earth term and the pulsar terms in the signal detected by PTAs (Equation 20). This is because the duration of the burst (by definition, less than the observational data span) is much less than 


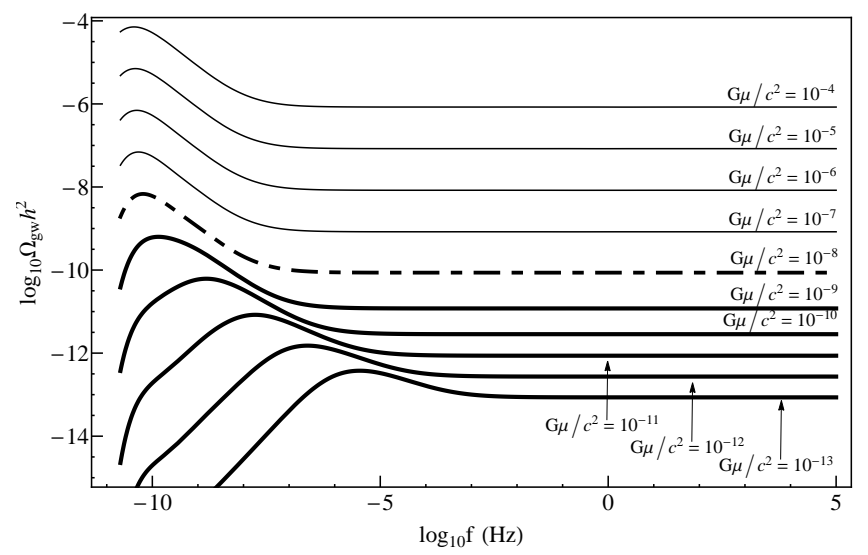

Fig. 16. Energy density spectrum for GWs emitted by cosmic strings as a function of the dimensionless string tension $G \mu / c^{2}$. Other parameters are held fixed at values $\alpha=10^{-7}, q=4 / 3$, $n_{*}=1$ and $p=1$. The dashed spectrum is for the critical point where $\Gamma G \mu / c^{2} \approx \alpha$; spectra above this are for large loops and spectra below are for small loops. (Ref. 111)

the light-time to the pulsars and so, except for a source in the same direction as a pulsar, the burst will occur at very different times in the Earth and pulsar terms. The pulsar term reflects the effect of the burst on the pulsar at a time $d / c$ before the burst arrives at the Earth, but the pulsar term is detected at a time $(d / c)(1+\cos \theta)$ later than the Earth term, where $d$ is the pulsar distance and $\theta$ is the angle between the pulsar and the GW propagation direction as seen from the Earth.

Figure 17 shows the GW waveform produced by the coalescence of two black holes in a coordinate system where all the signal is in $h_{+}$. The maximum amplitude of the waveform is about 0.1 in the time units of Figure 17, or about $0.1 c M T_{\odot} / D$ or $\sim 10^{-14} M_{9} / D_{G p c}$ where $M_{9} \equiv 10^{-9} M$ is the total system mass in solar units and $D_{G p c}$ is the (comoving) distance in Gpc. Although this is comparable to the strain sensitivities achieved by current PTAs for continuous GW signals (see 33.3 below), even for the largest SMBHs, the timescale of the burst, $\sim 200 M T_{\odot}$ is only of order 10 days and the period of the oscillation is about an order of magnitude less than that. Not only is this too short to be resolved by any existing PTA, but the sensitivity over this short interval would be much less than that achieved for $\mathrm{CW}$ signals integrated over the entire data span. Space-based laser interferometer systems such as the planned eLISA $A^{3}$ have the potential to directly detect these bursts.

However, Figure 17 also shows another effect known as GW "memory" which is potentially detectable with pulsar timing. During the coalescence event, a nonoscillatory component to the gravitational strain builds up, so that at the end of the "ring-down" phase, the strain has a permanent offset from the pre-coalescence 


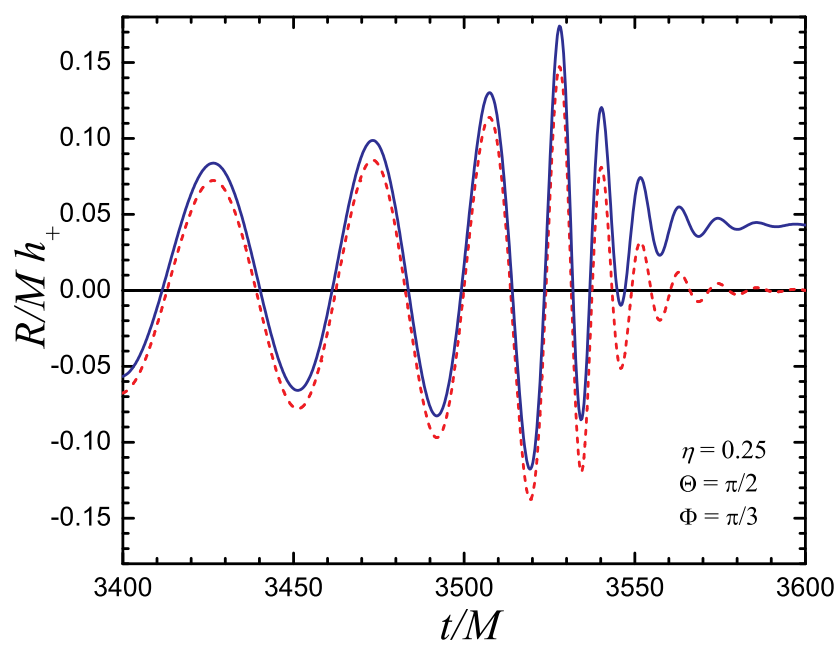

Fig. 17. Gravitational waveform resulting from the coalescence of two equal-mass black holes (reduced mass ratio $\eta=0.25$ ) with total mass $M$ at distance $R$. Both $M$ and $R$ are expressed in time units; the conversions to conventional units are $M \rightarrow M T_{\odot}$ and $R \rightarrow R / c . \Theta$ and $\Phi$ are assumed source directions. The dashed line is the predicted waveform if the gravitational memory effect is ignored. (Ref. 44)

value. The amplitude of the memory effect is

$$
h_{m}=\frac{\epsilon \eta M T_{\odot}}{D / c} \approx 10^{-15} \frac{M_{9}}{D_{9}}
$$

where $\epsilon \approx 0.07$ is the mass fraction contributing to the memory effect and $\eta$ is the reduced mass fraction, 0.25 for equal-mass binary components. $\underline{4430}$

This step change in $h$ produces a step change in the observed pulse frequency $\Delta \nu / \nu=h_{m}$, i.e., a "glitch". This glitch persists until it is reversed by the pulsar term at a time $(d / c)(1+\cos \theta)$ later. In a PTA, these reversals will occur at different times for different pulsars. Of course, it is also possible that a GW memory jump could be detected in the pulsar terms, but there it may be confused with a real glitch in the intrinsic pulse frequency, whereas in the Earth term there is a correlation in the effect on different pulsars. However, glitches in MSPs are rare (only one very small glitch detected so far: Ref. 29). Also, real pulsar glitches are generally spinups, whereas a GW-memory jump may be of either sign, depending on pulsar-source angle. Therefore, as Cordes and Jenet ${ }^{30}$ have discussed, the pulsar terms may give an improved probability of detection.

Black-hole binary systems with circular orbits emit GW at the second harmonic of the orbital frequency, i.e., $f=2 f_{b}$. For eccentric orbits, the GW emission becomes more burst-like as the accelerations and hence GW power are greatest around periastron when the two black holes are closest together ${ }^{102}$ In the spectral domain, power spreads to higher harmonics and also to the fundamental frequency $f_{b}$. In the gravitational-decay phase of evolution, when energy loss is dominated by GW emis- 
sion, the orbit tends to circularise ${ }^{8}$ However, at earlier phases of the orbital decay when three-body stellar interactions or interaction with a gaseous disk surrounding the binary system are important, the eccentricity may grow. Stellar three-body interactions can result in orbital decay through dynamical friction but probably result in a modest increase in the eccentricity of the black-hole binary system (e.g., Ref. 90 ). However, Roedig et al ${ }^{110}$ find that initially mildly eccentric binary systems decaying through interaction with a gaseous disk evolve toward a limiting eccentricity in the $0.6-0.8$ range. In a regime of frequent mergers it is even possible that a black-hole triplet could form ${ }^{4}$ and, in this case, eccentricities as high as 0.99 could exist.

Finn and Lommen ${ }^{47}$ have investigated the GW emission and the resulting timing residuals from a close parabolic encounter of two massive black holes. As Figure 18 shows, an encounter of two $10^{9} M_{\odot}$ black holes located at a distance of $15 \mathrm{Mpc}$ with a minimum separation of 0.02 pc produces a GW burst of duration about 1 year and maximum GW strain $\sim 10^{-13}$. This results in potentially detectable PTA timing residuals of about $1 \mu$ s amplitude with the same timescale. Unfortunately, the probability of having such a close encounter of two SMBHs in the local universe within PTA observational data spans is not high.
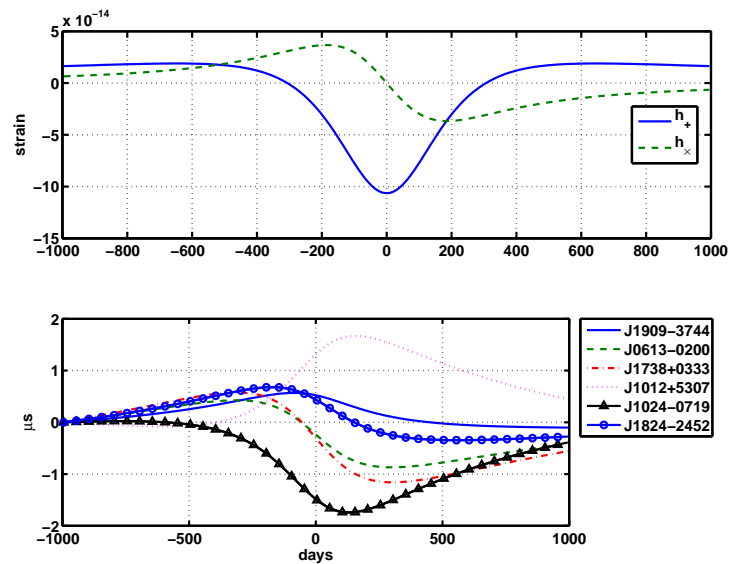

Fig. 18. The upper plot shows the gravitational waveforms in the $h_{+}$and $h_{-}$polarisations resulting from a parabolic encounter with an impact parameter of $0.02 \mathrm{pc}$ of two $10^{9} M_{\odot}$ black holes located at a distance of $15 \mathrm{Mpc}$ in the direction of the Virgo Cluster. The lower plot shows the resulting timing residuals for several PTA pulsars. (Ref. 47)

Cosmic strings are another potential source of GW burst emission. They can radiate over a wide frequency range with a huge range of possible amplitudes and timescales (cf., Figure 16) depending on the detailed mechanism invoked (loops, cusps, short strings, etc.) and the very wide (virtually unlimited) param-

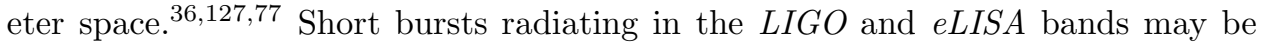


frequent and unresolved, producing a GWB at these frequencies. However, bursts with longer timescales, producing radiation in the nanohertz band, are also possible but are likely to be extremely rare ${ }^{\sqrt{66}}$ Consequently, while in principle such bursts could be detected, in practice it is unlikely that PTAs will be able to significantly constrain models for GW burst emission from cosmic strings.

\subsection{Pulsar Timing Arrays and Current Results}

In this section we first describe the three main PTAs currently operating world-wide: the European Pulsar Timing Array (EPTA), the North American pulsar timing array (NANOGrav) and the Parkes Pulsar Timing Array (PPTA), and the collaboration between them, the International Pulsar Timing Array (IPTA). PTAs have many possible applications such as establishing a pulsar-based timescale,, 57 investigating the accuracy of solar-system ephemerides, $\stackrel{26}{26}$ and investigating the properties of the pulsars themselves (e.g., Refs 156, 118) and of the intervening interstellar medium (e.g., Ref. 67). However, here we concentrate on what is undoubtedly their primary scientific goal, the direct detection of gravitational waves. Unfortunately, in common with other GW detection efforts around the world, PTAs have so far only been able to place limits on the strength of signals from potential GW sources. However, these limits are now beginning to seriously constrain the astrophysical source models and the assumptions that go into them and hence have implications that go far beyond the GW studies themselves.

\subsubsection{Existing PTAs}

The EPTA uses five large radio-telescopes in Europe, the Effelsberg 100-m telescope in Germany, the Nançay Radio Telescope in France (95-m equivalent area), the Westerbork Synthesis Radio Telescope in the Netherlands (similar effective area to the Nançay telescope), the 76-m Lovell Telescope at Jodrell Bank in England and the recently completed 64-m Sardinia Radio Telescope in Italy, to observe about 40 MSPs with a cadence of between a few days and 30 days for different pulsars. ${ }^{73}$ Different telescopes observe at different frequencies in the range $0.3-2.6 \mathrm{GHz}$, but all are instrumented at $1.4 \mathrm{GHz}$. Normally the five telescopes observe independently, but in a project known as the "Large European Array for Pulsars" (LEAP) 1.4 GHz signals over a bandwidth of $128 \mathrm{MHz}$ from the five telescopes can be summed coherently to form a 194-m equivalent diameter radio telescope. The different telescopes use different signal-processing systems, either digital filterbanks or coherent dedisperion systems or both.

NANOGrav makes use of the 300-m Arecibo radio telescope in Puerto Rico and the $100-\mathrm{m}$ Green Bank Telescope $(G B T)$ in West Virginia. ${ }^{87}$ A sample of about 36 pulsars is observed, typically at 3 -week intervals. At Arecibo, observations are made in bands centred at $430 \mathrm{MHz}$ and $1410 \mathrm{MHz}$, whereas at the GBT, the observed bands are centred at $820 \mathrm{MHz}$ and $1500 \mathrm{MHz}$. Currently both radio telescopes use

coherent dedispersion systems with bandwidths up to $800 \mathrm{MHz}$, but in the past a 
range of filterbank and coherent dedispersion systems with more limited bandwidths have been used.

As the name suggests, the PPTA uses the Parkes 64-m radio telescope located in New South Wales, Australia. A sample of 22 pulsars is currently being observed with regular observations at $2-3$ week intervals in three bands around $730 \mathrm{MHz}, 1400$ $\mathrm{MHz}$ and $3100 \mathrm{MHz}$ respectively ${ }^{[6 / 56}$ Coherent dedispersion systems are used at $730 \mathrm{MHz}$ and $1400 \mathrm{MHz}$ with bandwidths up to $310 \mathrm{MHz}$ and digital filterbanks at $1400 \mathrm{MHz}$ and $3100 \mathrm{MHz}$ with bandwidths of $256 \mathrm{MHz}$ and $1024 \mathrm{MHz}$ respectively.

Data sets from all three PTAs have spans ranging from a few years up to about 20 years for different pulsars; three (including the original MSP, PSR B1937+21) have Arecibo data spans of nearly 30 years. The three PTAs together observe about 50 pulsars with some being observed by two or even all three of the PTAs. Their distribution on the sky is shown in Figure 19.

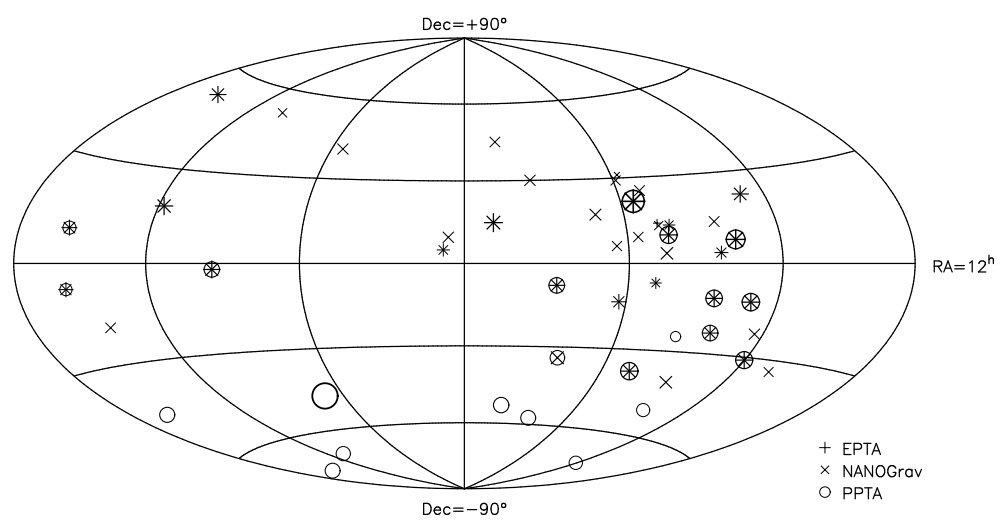

Fig. 19. Distribution on the sky of MSPs being timed by the three PTAs, with different symbols for each PTA. Right ascension increases to the left with $0^{\mathrm{h}}$ at the plot centre. The symbol size is related to the ratio $S_{1400} / P$, where $S_{1400}$ and $P$ are the pulsar $1400 \mathrm{MHz}$ flux density and pulse period respectively. (Ref. 85)

Given that the combined data set of the three PTAs contains a larger number of pulsars, improved observation cadence and greater frequency diversity than the data set of any one PTA, there is a strong motivation to combine all the available data sets to obtain maximum sensitivity for PTA scientific objectives. The International Pulsar Timing Array (IPTA) consortium was set up to facilitate progress toward this goal. $\frac{85}{5}$ The IPTA also arranges annual science meetings and student workshops and provides a forum for outreach programs and other activities related to PTA research.

\subsubsection{Limits on the nanohertz $G W$ background}

As discussed in Section 3.2.1. GWs from a cosmological distribution of SMBH binary systems are expected to contribute a very "red" signal to the spectrum of 
pulsar timing residuals. The expected signal from other GWB sources is similar. Consequently, long-term observations of a single pulsar with little or no detectable intrinsic timing irregularities can be used to place a limit on the strength of the GWB in the Galaxy. Of course, statistical limits can be improved by using data from several such pulsars. An early limit on the GWB at a frequency of $4.5 \mathrm{nHz}$ was set by Kaspi et al ${ }^{[66}$ using Arecibo observations of two MSPs, PSR B1855+09 and PSR B1937+21, with a $95 \%$ confidence limit on $\Omega_{G W} h^{2}$ of $6 \times 10^{-8}$, where $h=H_{0} / 100 \mathrm{~km} \mathrm{~s}^{-1}$.

Since the advent of the various PTA projects, both the quality and quantity of timing data sets has improved and a variety of analysis techniques have been employed to extract increasingly restrictive limits. Based on early PPTA data on seven pulsars combined with the Kaspi et al. PSR B1855+09 data set, Jenet et al ${ }^{64}$ used a "frequentist" approach with a statistic based on the amplitude of the low-frequency components in the power spectrum of the timing residuals to set a $95 \%$ confidence limit of about $2 \times 10^{-8}$ on $\Omega_{G W}$ at a GW frequency of $1 / 8 \mathrm{yr}$ or $4 \mathrm{nHz}$. From Equations 25 and 30 , this result is equivalent to a characteristic strain at frequency $1 / 1 \mathrm{yr}, A_{1 \mathrm{yr}} \approx 1.1 \times 10^{-14}$.

van Haasteren et al! ${ }^{138}$ analysed EPTA $1400-\mathrm{MHz}$ data sets for five MSPs with spans of $5-8$ years using a Bayesian analysis to place limits on the GWB amplitude as a function of its spectral index $\alpha$. For $\alpha=-2 / 3$, the derived limit at the $95 \%$ confidence level is $A_{1 \mathrm{yr}} \approx 6 \times 10^{-15}$, about a factor 1.8 better than the Jenet et al. ${ }^{64}$ limit.

NANOGrav multi-band data sets recorded between 2005 and 2010 for 17 MSPs were analysed by Demorest et al. $\frac{40}{0}$ Timing analyses taking into account timevarying dispersion delays and frequency-dependent pulse profiles were carried out to form sets of post-fit residuals and the corresponding covariance matrices for each pulsar. For most MSPs in the sample, no red noise signal was detectable in the post-fit residuals. Considering just the pulsar with the smallest post-fit residuals, PSR J1713+0747, and taking into account absorption of red noise by the timing fit, Demorest et al. obtained a $95 \%$ confidence limit for $A_{1 \mathrm{yr}}$ of $1.1 \times 10^{-14}$. A separate cross-correlation analysis weighted by the expected Hellings \& Downs function (Equation 221) across all the pulsars in the sample resulted in a somewhat better limit $A_{1 \mathrm{yr}} \approx 7 \times 10^{-15}$, although this limit was dominated by correlations with the two best pulsars in the sample, PSRs J1713+0747 and J1909-3744.

Based on PPTA and earlier Parkes timing observations made in three observing bands centred near $700 \mathrm{MHz}, 1400 \mathrm{MHz}$ and $3100 \mathrm{MHz}$ respectively with data spans of up to 17 years,$\sqrt[86]{ }$ together with the PSR B1855+09 archival Arecibo data, 66 Shannon et al $\frac{119}{[19}$ placed a limit of $1.3 \times 10^{-9}$ on $\Omega_{G W}$ at a GW frequency of 2.8 nHz. The corresponding limit on $A_{1 \mathrm{yr}}$ assuming a GW spectral index of $-2 / 3$ is $2.4 \times 10^{-15}$. This analysis, which included dispersion correction for the PPTA data sets and was based on the six best PPTA pulsars, used a statistical method similar to that of Jenet et al. 64 but included modelling of the red noise in the timing residuals. As shown in Figure 20, this limit rules out a model in which the growth 
of SMBH in galaxies is dominated by mergers 89 at the $91 \%$ confidence level, but is consistent with other models for galaxy and SMBH evolution where much of the SMBH growth is by accretion.

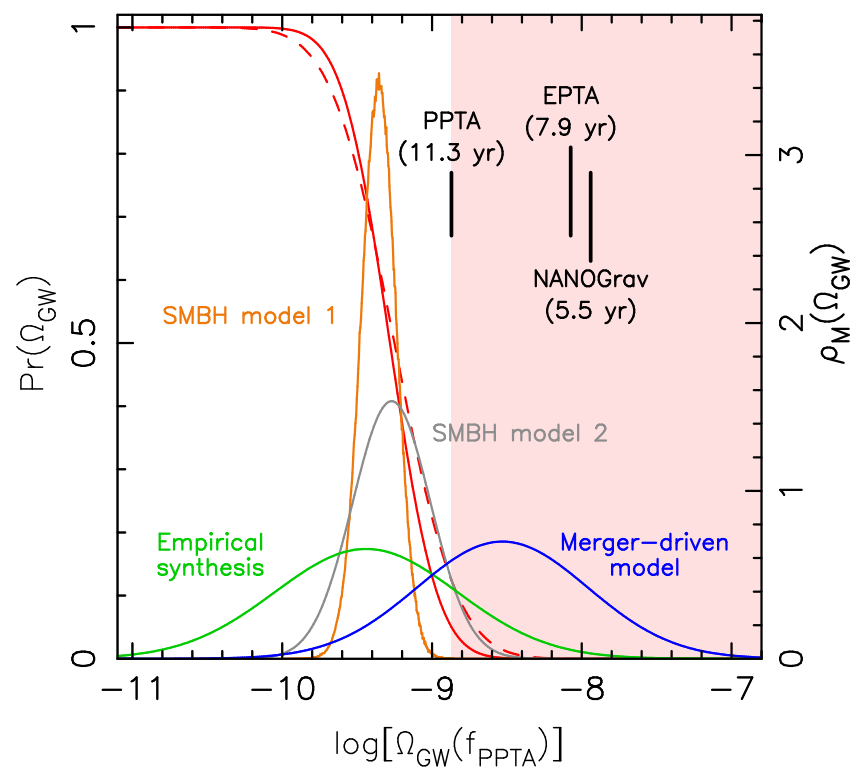

Fig. 20. Limits on the relative energy density of the GWB, $\Omega_{G W}$ at a GW frequency of $2.8 \mathrm{nHz}$ based on the PPTA data sets, together with predictions for $\Omega_{G W}$ based on several different models for the GWB ${ }^{119}$ The solid and dashed lines that are asymptotic to 1.0 at low $\Omega_{G W}$ show the probability $\operatorname{Pr}$ that a GWB signal of energy density $\Omega_{G W}$ can exist in the PPTA data sets, based on gaussian and non-gaussian GWB statistics respectively. The shaded region is ruled out with $95 \%$ confidence by the PPTA data. Corresponding limits from analysis of EPTA $A^{138}$ and NANOGrav data sets, scaled to $f_{G W}=2.8 \mathrm{nHz}$, are also shown. The gaussian curves show the probabilility density functions $\rho_{M}$ for the existence of a GWB with energy density $\Omega_{G W}$ based on a mergerdriven model for growth of SMBHs in galaxies, ${ }^{89}$ an empirical synthesis of observational constraints on SMBHs in galaxies, $\frac{113}{13}$ and based on the Millenium dark matter simulations $\frac{18}{18}$ together with semi-analytic models for growth of SMBHs in galaxies. (See Ref. 119 for more detail.)

As discussed in Section 3.2.2 topological defects in the early Universe are another potential source for the GWB. Figure 21 shows limits on the dimensionless string tension $G \mu / c^{2}$ as a function of loop size for various sets of other relevant parameters 111 The middle solid curves are limits based on the current EPTA data sets and the lower dashed curves are projections for LEAP data sets that coherently combine data from the EPTA telescopes. The upper dot-dashed line is a limit based on LIGO data! ${ }^{1}$ The current EPTA results give a conservative upper limit on the string tension of $5.3 \times 10^{-7}$. Lower limits can be obtained with more restricted assumptions about the string parameters (e.g., Refs 37, 64, 138). 


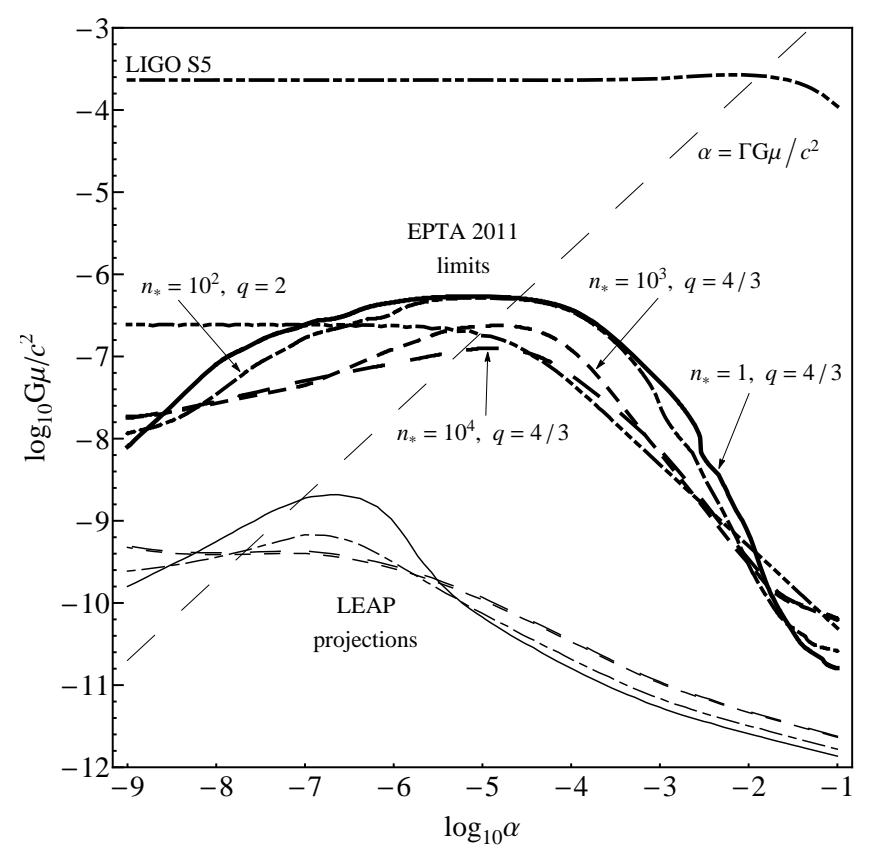

Fig. 21. Limits on cosmic string tension as a function of the loop size scale parameter $\alpha$ for different cutoff node numbers $n_{*}$ and intrinsic spectral slopes $q$ for the current EPTA limit on the energy density of the GWB (thick lines) and the projected sensitivity of the LEAP PTA (corresponding thin lines). The line with one long dash and three short dashes is an analytic approximation which is valid for large loops. The uppermost line is the $L I G O$ limit at $f=1 \mathrm{kHz}$. (Refs 111 1)

\subsubsection{Limits on $G W$ emission from individual black-hole binary systems}

For an isolated binary system at a luminosity distance $d_{L}$, the intrinsic GW strain amplitude is given by

$$
h_{0}=\frac{\left(G M_{c}\right)^{5 / 3}}{c^{4}} \frac{(\pi f)^{2 / 3}}{d_{L}}
$$

where $M_{c}$ is the binary chirp mass and $f=2 f_{b}$ is the GW frequency. The actual observed signal depends on the orbital orientation and phase as well as the GW polarisation angle. By averaging over these quantities, PTAs can set probabilistic limits for the strain amplitude as a function of $f$, both in a given direction and averaged over all directions (see, e.g., Refs 9, 158). In these analyses, there is assumed to be negligible evolution of $f$ over the data span and only the Earth term (cf. Equation 20) is considered; because of uncertainties in the pulsar distances, the pulsar terms cannot be added coherently and just contribute noise.

Figure 22 shows both sky-averaged upper limits and detection sensitivity for continuous-wave GW signals as a function of GW frequency based on the PPTA data set and using a frequentist analysis method ${ }^{158}$ The best limits and sensitivity are obtained for $\mathrm{GW}$ frequencies around $10^{-8} \mathrm{~Hz}$, where the upper limit on $h_{0}$ 
is about $1.1 \times 10^{-14}$. A similar analysis of the five-year NANOGrav data set by Arzoumanian et al ${ }^{9}$ using both frequentist and Bayesian analysis methods gave a somewhat higher sky-averaged upper limit of about $5 \times 10^{-14}$ at $10^{-8} \mathrm{~Hz}$. Because of the uneven sky distribution of PTA pulsars, there is quite a strong dependence of sensitivity on source direction. This is illustrated in Figure 23 which shows that sensitivity is greater toward the greatest concentration of PTA pulsars, roughly toward the Galactic Centre.

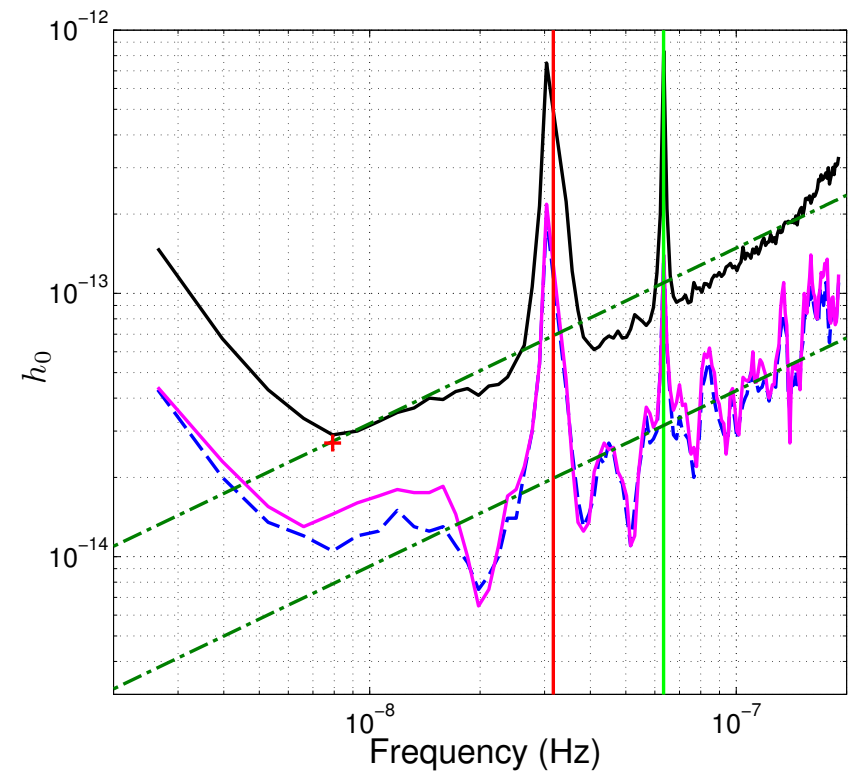

Fig. 22. Sky-averaged limits on the intrinsic GW strain amplitude $h_{0}$ as a function of GW frequency $f$ based on the PPTA data sets. The lower curves represent the largest GW signal (with a false-alarm probability of $1 \%$ ) that could be present in the real PPTA data (dashed line) and a simulated data set (solid line). The upper solid line gives the sensitivity of the PPTA to a continuous-wave source, i.e., the minimum signal that could be detected with $95 \%$ probability. The upper limits and sensitivities are higher at frequencies of $1 / 1 \mathrm{yr}$ and $1 / 6$ months as these frequencies are absorbed by the timing fits for position and parallax respectively. The sloping dotdashed lines are the expected signal levels for a SMBH binary systems with $M_{c}=10^{10} \mathrm{M}_{\odot}$ and distance $400 \mathrm{Mpc}$ (upper line) and $M_{c}=10^{9} \mathrm{M}_{\odot}$ and distance $30 \mathrm{Mpc}$ (lower line). (Ref. 158)

Figure 22 also shows that we can effectively rule out the existence of SMBH binary systems with $M_{c}=10^{9} \mathrm{M}_{\odot}$ and orbital frequencies around $10^{-8} \mathrm{~Hz}$ at distances closer than $30 \mathrm{Mpc}$. Similarly, a system with $M_{c}=10^{10} \mathrm{M}_{\odot}$ at a distance of $400 \mathrm{Mpc}$ should be detectable. Unfortunately, as Figure 23 shows, the nearby galaxy clusters such as Virgo, Coma and Fornax are all in regions of relatively low sensitivity for the PPTA (and other PTAs), so the effective limits for these clusters are a factor of a few higher. It is unlikely that such massive binary systems exist in these clusters. More generally, limits can also be placed on the SMBH binary 


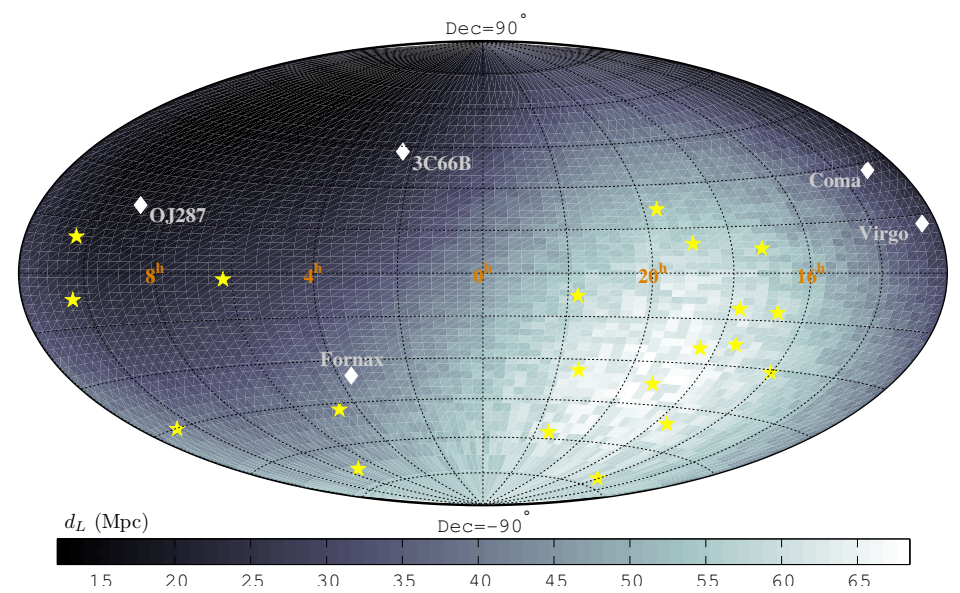

Fig. 23. Sky distribution of the luminosity distance $d_{L}$ to which a binary system with chirp mass $M_{c}=10^{9} \mathrm{M}_{\odot}$ radiating at $10^{-8} \mathrm{~Hz}$ could be detected. The stars indicate the positions of the 20 PPTA pulsars and the diamonds are potential sources of GW continuous-wave emission. (Ref.[158)

coalescence rate in the nearby Universe $(z \lesssim 0.1)$. Based on the PPTA results, Zhu et al ${ }^{[58}$ place a $95 \%$ confidence limit of $4 \times 10^{-3}\left(10^{10} \mathrm{M}_{\odot} / M_{c}\right)^{10 / 3} \mathrm{Mpc}^{-3} \mathrm{Gyr}^{-1}$ on the coalescence rate. This limit is about two orders of magnitude above current estimates of the galaxy merger rate in the local Universe (cf. Ref. 9).

These rate estimates are based on the orientation-averaged and sky-averaged amplitudes. It is of course possible that a favourably oriented and located SMBH binary system in the late stages of coalescence could exist. On the other hand, the estimates are based on circular binary orbits and, as discussed in Sections 3.2.1 and 3.2 .3 above, short-period SMBH binaries may have significant eccentricity which reduces the GW power at the fundamental frequency $f=2 f_{b}$ and hence the detectability of such systems. On balance, it seems unlikely that GW from an individual coalescing SMBH binary system will be detected with the current generation of PTAs.

\subsection{Future Prospects}

PTAs have now achieved data spans and ToA precisions that would allow detection of the GWB predicted by some models for the evolution of galaxies and the SMBHs at their core (see, e.g., Ref. [119). Up to now, no detections have been made. While this is disappointing from the point of view of GW astrophysics, it is starting to have important implications for galaxy and SMBH evolution models and to rule out some scenarios. It also implies that PTAs are close to detecting the GWB if current predictions for its amplitude are correct.

Siemens et al ${ }^{126}$ have considered the sensitivity of an idealised PTA to a GWB. At low signal levels, when the lowest signal frequencies are below the white noise 
level, the detection signal-to-noise ratio $(\mathrm{S} / \mathrm{N})$ is

$$
\langle\rho\rangle \propto M c \frac{A_{1 \mathrm{yr}}^{2}}{\sigma^{2}} T^{\beta}
$$

where $M$ is the number of pulsars in the array, $c$ is the observing cadence (frequency of observations), $A_{1 \mathrm{yr}}$ is the GWB amplitude (Equation 30), $\sigma$ is the rms level of the white timing noise, $T$ is the observing data span and $\beta$ is the inverse spectral index of the GWB signal in the timing residuals, taken to be $13 / 3$ (Equation 31). In the detection regime where the GWB signal exceeds the white noise level, the $\mathrm{S} / \mathrm{N}$ is

$$
\langle\rho\rangle \propto M\left(\frac{\sqrt{c} A_{1 \mathrm{yr}}}{\sigma}\right)^{1 / \beta} T^{1 / 2} .
$$

Consequently, in the pre-detection regime, the $\mathrm{S} / \mathrm{N}$ increases rapidly with increased observing cadence and data span and decreased timing noise, but has a much weaker dependence on these parameters in the strong signal regime. The reason for this is that noise from the uncorrelated pulsar term, which is also proportional to $A_{1 \mathrm{yr}}$, dominates over the white "receiver" noise, greatly modifying the statistical behaviour. Importantly though, in both regimes, the $\mathrm{S} / \mathrm{N}$ is proportional to $M$, the number of pulsars in the PTA. Figure 24 illustrates these dependencies for a range of plausible future PTAs.

As Siemens et al ${ }^{126}$ point out, if $A_{1 \mathrm{yr}} \sim 10^{-15}$, current PTAs are already in the "strong signal" regime. This means that increasing the observing data spans and cadence or decreasing ToA uncertainties has limited effect on the $\mathrm{S} / \mathrm{N}$ of a potential detection. Increasing the number of pulsars in the PTA is a much more cost-effective way to increase detection sensitivity. This fact provides much of the motivation to combine data from existing PTAs to form the International Pulsar Timing Array (IPTA). In the future, the Chinese FAST radio telescope ${ }^{94}$ and the $S K A^{24}$ will provide a large increase in radiometer sensitivity compared to existing instruments. The discussion above shows that this increased sensitivity will be best employed in increasing the number of (weaker) pulsars that are timed, rather than improving the ToA precision on the stronger existing PTA pulsars. Considerations of "jitter noise" in ToAs resulting from shape variations in individual pulses ${ }^{\sqrt[118]{ }}$ lead to the same conclusion.

While direct detection of the GWB would be enormously exciting and significant, there is no doubt that direct detection of GW from individual SMBH binary systems is potentially much more interesting from an astrophysical perspective. It opens up the possibility of identifying a GW source with source or region identified through electromagnetic-wave (radio, optical, X-ray or $\gamma$-ray) emission and the advent of "multi-messenger" astronomy (e.g., Refs 78, 114, 22). For example, many active galactic nuclei (AGNs) show evidence of a close binary SMBH at their core. Examples of this include X-shaped radio lobes (e.g., Ref. 157), double-peaked or variable emission lines from the core region (e.g., Ref. 124), quasi-periodic modula- 


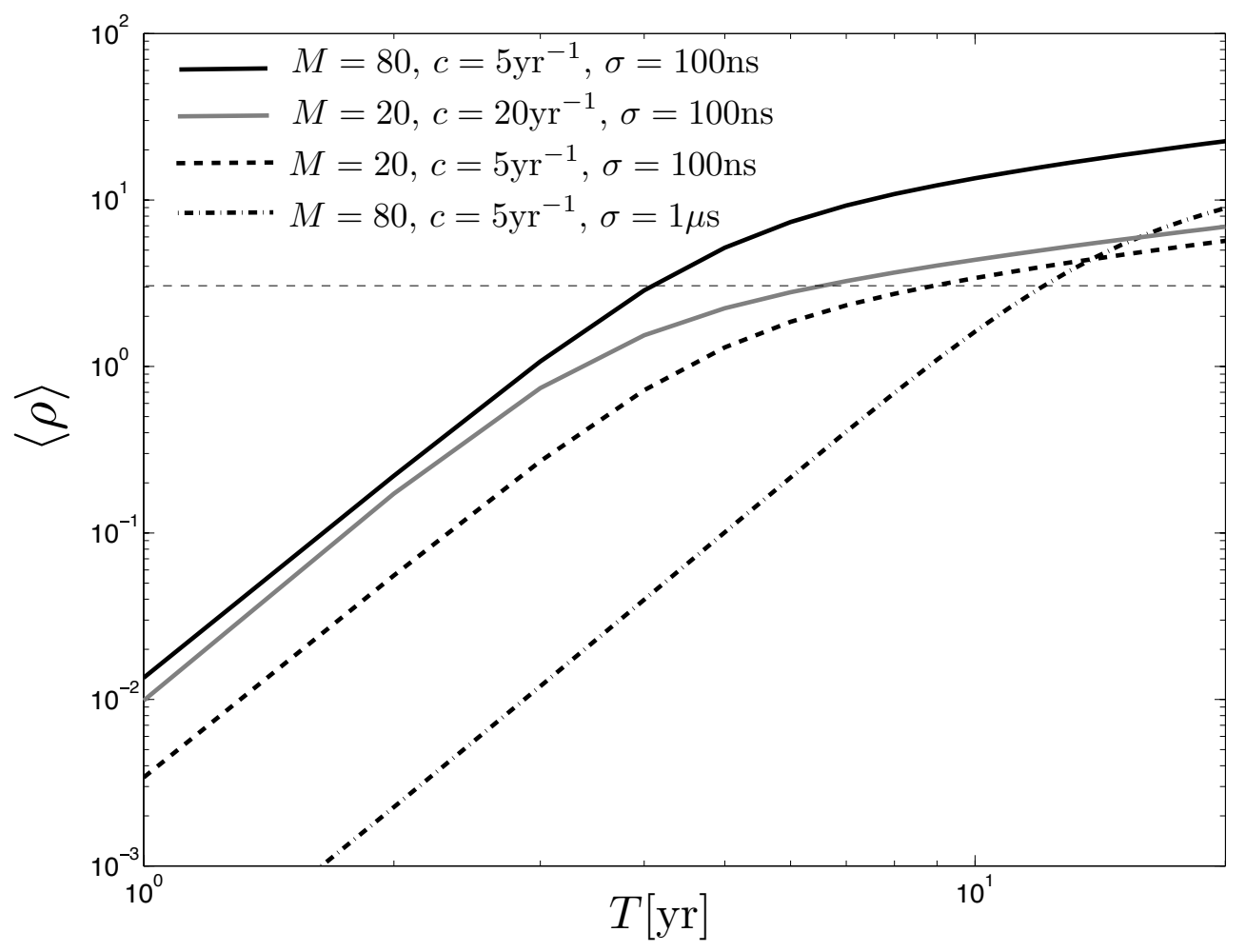

Fig. 24. Detection S/N for a GWB as a function of PTA data span for four different, but plausible, future PTAs. See text for the meaning of the PTA parameters. (Ref. 126)

tion of core radio (e.g., Ref. 137) or X-ray emission (e.g., Ref. 114, 79), and direct imaging of double AGN (e.g., Ref. 109).

With one exception, existing PTA systems do not have sufficient sensitivity to detect these potential GW sources. The exception is the claimed SMBH binary system identified by VLBI astrometry of the nearby quasar $3 \mathrm{C} 66 \mathrm{~B}^{133}$ which was effectively ruled out by pulsar timing observations ${ }^{65}$ Future PTAs including FAST and the $S K A$ will have much increased sensitivity, making searches for other GW candidate sources potentially more productive.

Similarly, although blind searches for continuous-wave GW signals in current PTA data sets have a low probability of successful detection, in future this should not be the case. The possibility of identification of a source galaxy or AGN then depends critically on the accuracy of the position determination for the GW source. When only detection of the "Earth term" is considered, the accuracy is at best many tens of square degrees (e.g., Ref. 158) containing thousands if not millions of galaxies. Only a correlation of the GW signal with a modulation of some property 
of a galaxy core (e.g., intensity or velocity) would establish an identification.

The situation changes dramatically if the pulsar terms can be added coherently with the Earth term (cf. Equation 20). Each Earth - pulsar system then forms an interferometer with baseline $d$ and fringe spacing $\sim \lambda_{G W} /[2 \pi d(1+\gamma)]$, where $d$ is the pulsar distance, $\lambda_{G W}$ is the GW wavelength and $\gamma$ is the direction cosine between the pulsar direction and the GW propagation direction. ${ }^{78 \mid 19}$ Positional accuracies are roughly the fringe spacing divided by the $\mathrm{S} / \mathrm{N}$ of the GW detection. Since $d$ is typically $>10^{3}$ light-years and $\lambda_{G W}$ is a few light-years, sub-arc-minute positional accuracies are possible for the stronger sources. However, to achieve the coherent summation, the distance to the pulsars must be known to better than $\lambda_{G W} /(1+\gamma)$, i.e., about $1 \mathrm{pc}$ unless the GW source is nearly aligned with the pulsar. Currently, only one PTA pulsar, PSR J0437-4715, has a distance known to this accuracy, measured through VLBI astrometry ${ }^{39}$ but in the $S K A$ era this will change. As Boyle and Pen ${ }^{19}$ point out, with a high density of PTA pulsars on the sky, advantage can be taken of the $(1+\gamma)$ factor and so pulsars with less precisely determined distances located in the general direction of the GW source could be useful. In this situation, PTAs would not be confusion-limited and, in principle, many individual GW sources could be identified. With a high enough number of PTA pulsars (say 1000 or more) it may be possible to localise a binary GW source by the quadrupolar pattern of timing residuals in pulsars surrounding the source, even if the pulsar distances are poorly determined.

\section{Summary and Conclusions}

Nature has been very kind in providing us with a set of near-perfect celestial clocks, many in situations of rapidly varying gravitational accelerations. Not only are these celestial clocks, known as pulsars, precise time-keepers, they are also exceedingly compact. This enables them to be treated as point masses in theoretical analyses of their motion and also permits tests in the regime of strong gravitational fields. These qualities result in a very wide range of applications for pulsar time-keeping, most importantly, at least in the context of this review, to investigations of relativistic gravitation.

Observations of double-neutron-star systems, wide circular pulsar - white-dwarf systems and even isolated pulsars have been used to test the accuracy of gravitational theories. Remarkably, general relativity (GR) is unscathed by all of these tests and hence remains the most viable theory of gravitation. Pulsar timing has provided the strongest available limits on at least six parameters describing deviations from GR. Continued and improved pulsar timing measurements, especially with new and highly sensitive radio telescopes such as FAST and the $S K A$, will both improve on

these limits and enable new and different tests of relativistic gravity. They may even demonstrate a failure of GR to adequately account for the observations, leading to new or modified theories of gravitation.

Continuing and new searches for previously unknown pulsars, especially with 
$F A S T$ and the $S K A$, will not only increase the number of pulsars that can be used in tests of relativistic gravity. They will also turn up new and exciting classes of object such as the recently discovered triple system, PSR J0337+1715. Such discoveries enrich the investigations of relativistic astrophysics that can be undertaken with pulsars.

One of the outstanding goals of current astronomy and astrophysics is the direct detection and study of gravitational waves (GWs). Pulsar timing arrays (PTAs) provide a viable mechanism for detection of GWs with frequencies in the nanohertz range. They therefore complement other existing or planned instruments such as the laser-interferometer systems LIGO and eLISA which are sensitive to GWs at frequencies of around $100 \mathrm{~Hz}$ and millihertz respectively. The most probable sources for GW detection by PTAs are binary super-massive black holes in the cores of distant galaxies. These produce an unresolved background of GWs that is potentially detectable, but there may also be individual binary systems that could be detected by PTAs.

There are currently three major PTAs operating, one each in Europe (EPTA), North America (NANOGrav) and Australia (PPTA). Up to now, no GWs have been detected by PTAs (or other GW detection systems) so the direct detection of GWs remains a goal. However, recent limits on the GW background are placing significant constraints on existing models for galaxy mergers over cosmological time and the formation and evolution of super-massive black holes in the cores of these galaxies. For example, a model in which black-hole growth is dominated by mergers is essentially ruled out.

The sensitivity of PTAs to GWs is a function of several factors including the precision of the pulse arrival-time measurements, the data span of the PTA observations and the cadence or frequency of observations within this data span. However the most important single factor is the number of pulsars in the PTA. Of course, these pulsars must meet certain timing-precision and period-stability criteria to usefully contribute to a PTA. There are two main approaches to increasing the number of pulsars. First, existing data sets can be combined to form a single PTA - this is the goal of the International Pulsar Timing Array project. Second, searches can be undertaken to increase the number of known pulsars suitable for PTA projects. With FAST and the SKA it is possible that hundreds of MSPs that are suitable for PTA projects will be both discovered and subsequently timed to high precision. This will surely lead to the detection of GWs and to detailed investigations of both the GWs themselves and the sources that generate them.

\section{Acknowledgments}

I thank my many colleagues in the pulsar community for their insights and hard work that have led to the results discussed in this review. In particular, I thank Paulo Freire, Matthew Kerr and Norbert Wex for helpful comments on earlier versions of the manuscript and Sydney Chamberlin, Sotiris Sanidas and Xingjiang Zhu for 
producing revised versions of figures from their papers. I also thank the CSIRO and the Australian Research Council for supporting my research over the years and, in particular, CSIRO Astronomy and Space Science for their continued support.

\section{References}

1. Abbott, B. P., Abbott, R., Acernese, F., Adhikari, R., Ajith, P., Allen, B., Allen, G., Alshourbagy, M., et al., The LIGO Collaboration and The Virgo Collaboration (2009a). An upper limit on the stochastic gravitational-wave background of cosmological origin, Nature 460, pp. 990-994.

2. Abbott, B. P., Abbott, R., Adhikari, R., Ajith, P., Allen, B., Allen, G., Amin, R. S., Anderson, S. B., et al. (2009b). LIGO: the Laser Interferometer Gravitational-Wave Observatory, Rep. Prog. Phys. 72, p. 076901.

3. Amaro-Seoane, P., Aoudia, S., Babak, S., Binétruy, P., Berti, E., Bohé, A., Caprini, C., Colpi, M., Cornish, N. J., et al. (2012). Low-frequency gravitational-wave science with eLISA/NGO, Class. Quant. Grav. 29, 12, 124016.

4. Amaro-Seoane, P., Sesana, A., Hoffman, L., Benacquista, M., Eichhorn, C., Makino, J. and Spurzem, R. (2010). Triplets of supermassive black holes: astrophysics, gravitational waves and detection, MNRAS 402, pp. 2308-2320.

5. Anholm, M., Ballmer, S., Creighton, J. D. E., Price, L. R. and Siemens, X. (2009). Optimal strategies for gravitational wave stochastic background searches in pulsar timing data, Phys. Rev. D 79, 8, p. 084030.

6. Antoniadis, J., Freire, P. C. C., Wex, N., Tauris, T. M., Lynch, R. S., van Kerkwijk, M. H., Kramer, M., Bassa, C., et al. (2013). A Massive Pulsar in a Compact Relativistic Binary, Science 340, p. 448.

7. Antoniadis, J., van Kerkwijk, M. H., Koester, D., Freire, P. C. C., Wex, N., Tauris, T. M., Kramer, M. and Bassa, C. G. (2012). The relativistic pulsar-white dwarf binary PSR J1738+0333 - I. Mass determination and evolutionary history, MNRAS 423, pp. 3316-3327.

8. Armitage, P. J. and Natarajan, P. (2005). Eccentricity of Supermassive Black Hole Binaries Coalescing from Gas-rich Mergers ApJ 634, pp. 921-927.

9. Arzoumanian, Z., Brazier, A., Burke-Spolaor, S., Chamberlin, S. J., Chatterjee, S., Cordes, J. M., Demorest, P. B., Deng, X., et al. (2014). Gravitational Waves from Individual Supermassive Black Hole Binaries in Circular Orbits: Limits from the North American Nanohertz Observatory for Gravitational Waves ApJ 794, 141.

10. Bekenstein, J. D. (2004). Relativistic gravitation theory for the modified Newtonian dynamics paradigm, Phys. Rev. D 70, 8, 083509.

11. Bell, J. F. (1996). A tighter constraint on post-Newtonian gravity using millisecond pulsars, ApJ 462, p. 287.

12. Bell, J. F. and Bailes, M. (1996). A new method for obtaining binary pulsar distances and its implications for tests of general relativity. ApJ 456, pp. L33-L36.

13. Bell, J. F. and Damour, T. (1996). A new test of conservation laws and Lorentz invariance in relativistic gravity, Class. Quant. Grav. 13, pp. 3121-3127.

14. Bertotti, B., Iess, L. and Tortora, P. (2003). A test of general relativity using radio links with the Cassini spacecraft, Nature 425, pp. 374-376.

15. Bhat, N. D. R., Bailes, M. and Verbiest, J. P. W. (2008). Gravitational-radiation losses from the pulsar white-dwarf binary PSR J1141 6545, Phys. Rev. D 77, 12, pp. 124017.

16. Barker, B. M. and O'Connell, R. F. (1975). Gravitational two-body problem with arbitrary masses, spins, and quadrupole moments, Phys. Rev. D 12, pp. 329-335. 
17. Bhattacharya, D. and van den Heuvel, E. P. J. (1991). Formation and evolution of binary and millisecond radio pulsars, Phys. Rep. 203, pp. 1-124.

18. Boylan-Kolchin, M., Springel, V., White, S. D. M., Jenkins, A. and Lemson, G. (2009). Resolving cosmic structure formation with the Millennium-II Simulation, MNRAS 398, pp. 1150-1164.

19. Boyle, L. and Pen, U.-L. (2012). Pulsar timing arrays as imaging gravitational wave telescopes: Angular resolution and source (de)confusion Phys. Rev. D 86, 12, 124028.

20. Breton, R. P., Kaspi, V. M., Kramer, M., McLaughlin, M. A., Lyutikov, M., Ransom, S. M., Stairs, I. H., Ferdman, R. D., Camilo, F. and Possenti, A. (2008). Relativistic Spin Precession in the Double Pulsar, Science 321, pp. 104.

21. Burgay, M., D'Amico, N., Possenti, A., Manchester, R. N., Lyne, A. G., Joshi, B. C., McLaughlin, M. A., Kramer, M., et al. (2003). An increased estimate of the merger rate of double neutron stars from observations of a highly relativistic system, Nature 426, pp. 531-533.

22. Burke-Spolaor, S. (2013). Multi-messenger approaches to binary supermassive black holes in the 'continuous-wave' regime, Class. Quant. Grav. 30, 22, 224013.

23. Caldwell, R. R. and Allen, B. (1992). Cosmological constraints on cosmic-string gravitational radiation, Phys. Rev. D 45, pp. 3447-3468.

24. Carilli, C. and Rawlings, S. (2004). Science with the Square Kilometre Array (New Astronomy Reviews, 48(11-12)).

25. Chamberlin, S. J. and Siemens, X. (2012). Stochastic backgrounds in alternative theories of gravity: Overlap reduction functions for pulsar timing arrays, Phys. Rev. D 85, 8, 082001.

26. Champion, D. J., Hobbs, G. B., Manchester, R. N., Edwards, R. T., Backer, D. C., Bailes, M., Bhat, N. D. R., Burke-Spolaor, S., Coles, W., Demorest, P. B., Ferdman, R. D., Folkner, et al. (2010). Measuring the Mass of Solar System Planets Using Pulsar Timing, ApJ 720, pp. L201-L205.

27. Chapon, D., Mayer, L. and Teyssier, R. (2013). Hydrodynamics of galaxy mergers with supermassive black holes: is there a last parsec problem?, MNRAS 429, pp. 3114-3122.

28. Clifton, T. and Weisberg, J. M. (2008). A Simple Model for Pulse Profiles from Precessing Pulsars, with Special Application to Relativistic Binary PSR B1913+16, ApJ 679, pp. 687-696.

29. Cognard, I. and Backer, D. C. (2004). A microglitch in the millisecond pulsar PSR B1821-24 in M28, ApJ 612, pp. L125-L127.

30. Cordes, J. M. and Jenet, F. A. (2012). Detecting Gravitational Wave Memory with Pulsar Timing, ApJ 752, 54.

31. Damour, T. and Esposito-Farèse, G. (1992). Tensor-multi-scalar theories of gravitation, Class. Quant. Grav. 9, pp. 2093-2176.

32. Damour, T. and Schäfer, G. (1988). Higher-order relativistic periastron advances and binary pulsars, Nuovo Cim. 101, p. 127.

33. Damour, T. and Schäfer, G. (1991). New tests of the strong equivalence principle using binary pulsar data, Phys. Rev. Lett. 66, p. 2549.

34. Damour, T. and Taylor, J. H. (1991). On the orbital period change of the binary pulsar PSR 1913+16, ApJ 366, pp. 501-511.

35. Damour, T. and Taylor, J. H. (1992). Strong-field tests of relativistic gravity and binary pulsars, Phys. Rev. D 45, pp. 1840-1868.

36. Damour, T. and Vilenkin, A. (2001). Gravitational wave bursts from cusps and kinks on cosmic strings, Phys. Rev. D 64, 6, p. 064008.

37. Damour, T. and Vilenkin, A. (2005). Gravitational radiation from cosmic (su- 
per)strings: Bursts, stochastic background, and observational windows, Phys. Rev. D 71, 6, p. 063510.

38. Deller, A. T., Bailes, M. and Tingay, S. J. (2009). Implications of a VLBI Distance to the Double Pulsar J0737-3039A/B, Science 323, pp. 1327.

39. Deller, A. T., Verbiest, J. P. W., Tingay, S. J. and Bailes, M. (2008). Extremely High Precision VLBI Astrometry of PSR J0437-4715 and Implications for Theories of Gravity, ApJ 685, pp. L67-L70.

40. Demorest, P. B., Ferdman, R. D., Gonzalez, M. E., Nice, D., Ransom, S., Stairs, I. H., Arzoumanian, Z., Brazier, A., Burke-Spolaor, S., Chamberlin, S. J., Cordes, J. M., Ellis, J., et al. (2013). Limits on the Stochastic Gravitational Wave Background from the North American Nanohertz Observatory for Gravitational Waves, ApJ 762, 94.

41. Desvignes, G., Kramer, M., Cognard, I., Kasian, L., van Leeuwen, J., Stairs, I. and Theureau, G. (2013). PSR J1906+0746: From relativistic spin-precession to beam modeling in J. van Leeuwen (ed.), Neutron Stars and Pulsars: Challenges and Opportunities after 80 Years, IAU Symposium 291 (Cambridge University Press, Cambridge), pp. 199-202.

42. Detweiler, S. (1979). Pulsar timing measurements and the search for gravitational waves, ApJ 234, p. 1100.

43. Einstein, A. (1915). Erklärung der Perihelbewegung des Merkur aus der allgemeinen Relativitätstheorie, Sitzungsberichte der Königlich Preußischen Akademie der Wissenschaften (Berlin), pp. 831-839.

44. Favata, M. (2009). Nonlinear Gravitational-Wave Memory from Binary Black Hole Mergers, ApJ 696, pp. L159-L162.

45. Ferdman, R. D., Stairs, I. H., Kramer, M., Breton, R. P., McLaughlin, M. A., Freire, P. C. C., Possenti, A., Stappers, B. W., et al. (2013). The Double Pulsar: Evidence for Neutron Star Formation without an Iron Core-collapse Supernova, ApJ 767, 85.

46. Ferdman, R. D., Stairs, I. H., Kramer, M., Janssen, G. H., Bassa, C. G., Stappers, B. W., Demorest, P. B., Cognard, I., et al. (2014). PSR J1756-2251: a pulsar with a low-mass neutron star companion, MNRAS 443, pp. 2183-2196.

47. Finn, L. S. and Lommen, A. N. (2010). Detection, Localization, and Characterization of Gravitational Wave Bursts in a Pulsar Timing Array ApJ 718, pp. 1400-1415.

48. Folkner, W. M., Williams, J. G. and Boggs, D. H. (2009). The planetary and lunar ephemeris DE 412, IPN Progress Report 42-178, NASA Jet Propulsion Laboratory.

49. Fonseca, E., Stairs, I. H. and Thorsett, S. E. (2014). A Comprehensive Study of Relativistic Gravity Using PSR B1534+12, ApJ 787, 82.

50. Freire, P. C. C., Bassa, C. G., Wex, N., Stairs, I. H., Champion, D. J., Ransom, S. M., Lazarus, P., Kaspi, V. M., et al. (2011). On the nature and evolution of the unique binary pulsar J1903+0327, MNRAS 412, pp. 2763-2780.

51. Freire, P. C. C., Kramer, M. and Wex, N. (2012). Tests of the universality of free fall for strongly self-gravitating bodies with radio pulsars, Class. Quant. Grav. 29, 18, 184007.

52. Freire, P. C. C., Wex, N., Esposito-Farèse, G., Verbiest, J. P. W., Bailes, M., Jacoby, B. A., Kramer, M., Stairs, I. H., et al. (2012). The relativistic pulsar-white dwarf binary PSR J1738+0333 - II. The most stringent test of scalar-tensor gravity, MNRAS 423, pp. 3328-3343.

53. Gonzalez, M. E., Stairs, I. H., Ferdman, R. D., Freire, P. C. C., Nice, D. J., Demorest, P. B., Ransom, S. M., Kramer, M., et al. (2011). High-precision Timing of Five Millisecond Pulsars: Space Velocities, Binary Evolution, and Equivalence Principles, ApJ 743, 102.

54. Guillemot, L., Johnson, T. J., Venter, C., Kerr, M., Pancrazi, B., Livingstone, M., 
Janssen, G. H., Jaroenjittichai, P., et al. (2012). Pulsed Gamma Rays from the Original Millisecond and Black Widow Pulsars: A Case for Caustic Radio Emission?, ApJ 744, 33 .

55. Hellings, R. W. and Downs, G. S. (1983). Upper limits on the isotropic gravitational radiation background from pulsar timing analysis, ApJ 265, p. L39.

56. Hobbs, G. (2013). The Parkes Pulsar Timing Array, Class. Quant. Grav. 30, 22, 224007.

57. Hobbs, G., Coles, W., Manchester, R. N., Keith, M. J., Shannon, R. M., Chen, D., Bailes, M., Bhat, N. D. R., et al. (2012). Development of a pulsar-based timescale MNRAS 427, pp. 2780-2787.

58. Hobbs, G., Jenet, F., Lee, K. J., Verbiest, J. P. W., Yardley, D., Manchester, R., Lommen, A., Coles, W., et al. (2009). TEMPO2: a new pulsar timing package - III. Gravitational wave simulation, MNRAS 394, pp. 1945-1955.

59. Hobbs, G., Lorimer, D. R., Lyne, A. G. and Kramer, M. (2005). A statistical study of 233 pulsar proper motions, MNRAS 360, pp. 974-992.

60. Hofmann, F., Müller, J. and Biskupek, L. (2010). Lunar laser ranging test of the Nordtvedt parameter and a possible variation in the gravitational constant, $A \mathscr{E} A$ $\mathbf{5 2 2}$, L5.

61. Hulse, R. A. and Taylor, J. H. (1975). Discovery of a pulsar in a binary system, ApJ 195, pp. L51-L53.

62. Jacoby, B. A., Cameron, P. B., Jenet, F. A., Anderson, S. B., Murty, R. N. and Kulkarni, S. R. (2006). Measurement of Orbital Decay in the Double Neutron Star Binary PSR B2127+11C, ApJ 644, pp. L113-L116.

63. Jaffe, A. H. and Backer, D. C. (2003). Gravitational waves probe the coalescence rate of massive black hole binaries, ApJ 583, pp. 616-631.

64. Jenet, F. A., Hobbs, G. B., van Straten, W., Manchester, R. N., Bailes, M., Verbiest, J. P. W., Edwards, R. T., Hotan, A. W., et al. (2006). Upper Bounds on the Low-Frequency Stochastic Gravitational Wave Background from Pulsar Timing Observations: Current Limits and Future Prospects, ApJ 653, pp. 1571-1576.

65. Jenet, F. A., Lommen, A., Larson, S. L. and Wen, L. (2004). Constraining the properties of supermassive black hole systems using pulsar timing: Application to 3C 66B, ApJ 606, pp. 799-803.

66. Kaspi, V. M., Taylor, J. H. and Ryba, M. (1994). High-precision timing of millisecond pulsars. III. Long-term monitoring of PSRs B1855+09 and B1937+21, ApJ 428, pp. $713-728$.

67. Keith, M. J., Coles, W., Shannon, R. M., Hobbs, G. B., Manchester, R. N., Bailes, M., Bhat, N. D. R., Burke-Spolaor, S., et al. (2013). Measurement and correction of variations in interstellar dispersion in high-precision pulsar timing $M N R A S$ 429, pp. 2161-2174.

68. Khan, F. M., Berentzen, I., Berczik, P., Just, A., Mayer, L., Nitadori, K. and Callegari, S. (2012). Formation and Hardening of Supermassive Black Hole Binaries in Minor Mergers of Disk Galaxies ApJ 756, 30.

69. Khan, F. M., Holley-Bockelmann, K., Berczik, P. and Just, A. (2013). Supermassive Black Hole Binary Evolution in Axisymmetric Galaxies: The Final Parsec Problem is Not a Problem, ApJ 773, 100.

70. Kiziltan, B., Kottas, A., De Yoreo, M. and Thorsett, S. E. (2013). The Neutron Star Mass Distribution, ApJ 778, 66.

71. Konopliv, A. S., Asmar, S. W., Folkner, W. M., Karatekin, Ö., Nunes, D. C., Smrekar, S. E., Yoder, C. F. and Zuber, M. T. (2011). Mars high resolution gravity fields from MRO, Mars seasonal gravity, and other dynamical parameters 211, pp. 401-428. 
72. Kramer, M. (1998). Determination of the geometry of the PSR B1913+16 system by geodetic precession, ApJ 509, pp. 856-860.

73. Kramer, M. and Champion, D. J. (2013). The European Pulsar Timing Array and the Large European Array for Pulsars, Class. Quant. Grav. 30, 22, 224009.

74. Kramer, M., Stairs, I. H., Manchester, R. N., McLaughlin, M. A., Lyne, A. G., Ferdman, R. D., Burgay, M., Lorimer, D. R., et al. (2006). Tests of General Relativity from Timing the Double Pulsar, Science 314, pp. 97-102.

75. Kramer, M. and Wex, N. (2009). TOPICAL REVIEW: The double pulsar system: a unique laboratory for gravity, Class. Quant. Grav. 26, 7, pp. 073001.

76. Lattimer, J. M. (2012). The Nuclear Equation of State and Neutron Star Masses, Ann. Rev. Nuclear \&S Particle Sci. 62, pp. 485-515.

77. Leblond, L., Shlaer, B. and Siemens, X. (2009). Gravitational waves from broken cosmic strings: The bursts and the beads, Phys. Rev. D 79, 12, 123519.

78. Lee, K. J., Wex, N., Kramer, M., Stappers, B. W., Bassa, C. G., Janssen, G. H., Karuppusamy, R. and Smits, R. (2011). Gravitational wave astronomy of single sources with a pulsar timing array, MNRAS 414, pp. 3251-3264.

79. Liu, F. K., Li, S. and Komossa, S. (2014). A Milliparsec Supermassive Black Hole Binary Candidate in the Galaxy SDSS J120136.02+300305.5, ApJ 786, 103.

80. Liu, K., Eatough, R. P., Wex, N. and Kramer, M. (2014). Pulsar-black hole binaries: prospects for new gravity tests with future radio telescopes, MNRAS 445, pp. 31153132.

81. Liu, K., Wex, N., Kramer, M., Cordes, J. M. and Lazio, T. J. W. (2012). Prospects for Probing the Spacetime of Sgr A* with Pulsars, ApJ 747, 1.

82. Lynch, R. S., Freire, P. C. C., Ransom, S. M. and Jacoby, B. A. (2012). The Timing of Nine Globular Cluster Pulsars, ApJ 745, 109.

83. Lyne, A. G., Burgay, M., Kramer, M., Possenti, A., Manchester, R. N., Camilo, F., McLaughlin, M. A., Lorimer, D. R., et al. (2004). A double-pulsar system: A rare laboratory for relativistic gravity and plasma physics, Science 303, pp. 1153-1157.

84. Lyutikov, M. and Thompson, C. (2005). Magnetospheric Eclipses in the Double Pulsar System PSR J0737-3039, ApJ 634, pp. 1223-1241.

85. Manchester, R. N. (2013). The International Pulsar Timing Array Class. Quant. Grav. 30, 22, 224010.

86. Manchester, R. N., Hobbs, G., Bailes, M., Coles, W. A., van Straten, W., Keith, M. J., Shannon, R. M., Bhat, N. D. R., et al. (2013). The Parkes Pulsar Timing Array Project, PASA 30, e017.

87. McLaughlin, M. A. (2013). The North American Nanohertz Observatory for Gravitational Waves, Class. Quant. Grav. 30, 22, 224008.

88. McLaughlin, M. A., Lyne, A. G., Lorimer, D. R., Possenti, A., Manchester, R. N., Camilo, F., Stairs, I. H., Kramer, M., et al. (2004). The Double Pulsar System J07373039: Modulation of A by B at Eclipse, ApJ 616, pp. L131-L134.

89. McWilliams, S. T., Ostriker, J. P. and Pretorius, F. (2014). Gravitational Waves and Stalled Satellites from Massive Galaxy Mergers at $\mathrm{z}<1$, ApJ 789, 156.

90. Merritt, D. and Milosavljević, M. (2005). Massive Black Hole Binary Evolution, Living Reviews in Relativity 8, p. 8.

91. Milgrom, M. (1983). A modification of the Newtonian dynamics as a possible alternative to the hidden mass hypothesis, ApJ 270, pp. 365-370.

92. Moore, C. J., Cole, R. H. and Berry, C. P. L. (2015). Gravitational-wave sensitivity curves, Class. Quant. Grav. 32, 1, 015014.

93. Müller, J., Williams, J. G. and Turyshev, S. G. (2008). Lunar Laser Ranging Contributions to Relativity and Geodesy in H. Dittus, C. Lammerzahl and S. G. Turyshev 
(eds.), Lasers, Clocks and Drag-Free Control: Exploration of Relativistic Gravity in Space, Astrophys. and Space Sci. Library, Vol. 349, p. 457.

94. Nan, R., Li, D., Jin, C., Wang, Q., Zhu, L., Zhu, W., Zhang, H., Yue, Y. and Qian, L. (2011). The Five-Hundred-Meter Aperture Spherical Radio Telescope (FAST) Project, Intnl J. of Mod. Phys. D 20, pp. 989-1024.

95. Ni, W.-T. (2010). Searches for the role of spin and polarization in gravity, Reports on Progress in Physics 73, 5, 056901.

96. Nice, D. J., Splaver, E. M., Stairs, I. H., Löhmer, O., Jessner, A., Kramer, M. and Cordes, J. M. (2005). A $2.1 M_{\odot}$ Pulsar Measured by Relativistic Orbital Decay, ApJ 634, pp. 1242-1249.

97. Nice, D. J., Stairs, I. H. and Kasian, L. E. (2008). Masses of Neutron Stars in Binary Pulsar Systems in C. Bassa, Z. Wang, A. Cumming and V. M. Kaspi (eds.), 40 Years of Pulsars: Millisecond Pulsars, Magnetars and More, Vol. 983 (American Institute of Physics, New York), pp. 453-458.

98. Nordtvedt, K. (1968). Testing relativity with laser ranging to the moon, Phys. Rev. 170, pp. 1186-1187.

99. Nordtvedt, K. (1987). Probing gravity to the second post-newtonian order and to one part in $10^{7}$ using the spin axis of the sun, ApJ 320, pp. 871-874.

100. Nordtvedt, K. (1990). $\dot{G} / G$ and a cosmological acceleration of gravitationally compact bodies, Phys. Rev. Lett. 65, pp. 953-956.

101. Perera, B. B. P., McLaughlin, M. A., Kramer, M., Stairs, I. H., Ferdman, R. D., Freire, P. C. C., Possenti, A., Breton, R. P., et al. (2010). The Evolution of PSR J0737-3039B and a Model for Relativistic Spin Precession, ApJ 721, pp. 1193-1205.

102. Peters, P. C. and Mathews, J. (1963). Gravitational Radiation from Point Masses in a Keplerian Orbit, Phys. Rev. 131, pp. 435-440.

103. Phinney, E. S. (2001). A Practical Theorem on Gravitational Wave Backgrounds, ArXiv:astro-ph/0108028 .

104. Ransom, S. M., Stairs, I. H., Archibald, A. M., Hessels, J. W. T., Kaplan, D. L., van Kerkwijk, M. H., Boyles, J., Deller, A. T., et al. (2014). A millisecond pulsar in a stellar triple system, Nature 505, pp. 520-524.

105. Ravi, V., Manchester, R. N. and Hobbs, G. (2010). Wide Radio Beams from $\gamma$-ray Pulsars, ApJ 716, pp. L85-L89.

106. Ravi, V., Wyithe, J. S. B., Hobbs, G., Shannon, R. M., Manchester, R. N., Yardley, D. R. B. and Keith, M. J. (2012). Does a "Stochastic" Background of Gravitational Waves Exist in the Pulsar Timing Band?, ApJ 761, 84.

107. Ravi, V., Wyithe, J. S. B., Shannon, R. M., Hobbs, G. and Manchester, R. N. (2014). Binary supermassive black hole environments diminish the gravitational wave signal in the pulsar timing band, MNRAS 442, pp. 56-68.

108. Rix, H.-W. and Bovy, J. (2013). The Milky Way's stellar disk. Mapping and modeling the Galactic disk, Astron. Asrophys. Rev. 21, 61.

109. Rodriguez, C., Taylor, G. B., Zavala, R. T., Peck, A. B., Pollack, L. K. and Romani, R. W. (2006). A Compact Supermassive Binary Black Hole System, ApJ 646, pp. 49-60.

110. Roedig, C., Dotti, M., Sesana, A., Cuadra, J. and Colpi, M. (2011). Limiting eccentricity of subparsec massive black hole binaries surrounded by self-gravitating gas discs, MNRAS 415, pp. 3033-3041.

111. Sanidas, S. A., Battye, R. A. and Stappers, B. W. (2012). Constraints on cosmic string tension imposed by the limit on the stochastic gravitational wave background from the European Pulsar Timing Array, Phys. Rev. D 85, 12, 122003.

112. Sazhin, M. V. (1978). Opportunities for detecting ultralong gravitational waves, Sov. 
Astron. 22, p. 36 .

113. Sesana, A. (2013). Systematic investigation of the expected gravitational wave signal from supermassive black hole binaries in the pulsar timing band, MNRAS 433, pp. L1-L5.

114. Sesana, A., Roedig, C., Reynolds, M. T. and Dotti, M. (2012). Multimessenger astronomy with pulsar timing and X-ray observations of massive black hole binaries, MNRAS 420, pp. 860-877.

115. Sesana, A., Vecchio, A. and Colacino, C. N. (2008). The stochastic gravitational-wave background from massive black hole binary systems: implications for observations with Pulsar Timing Arrays MNRAS 390, pp. 192-209.

116. Sesana, A., Vecchio, A. and Volonteri, M. (2009). Gravitational waves from resolvable massive black hole binary systems and observations with Pulsar Timing Arrays, MNRAS 394, pp. 2255-2265.

117. Shannon, R. M. and Cordes, J. M. (2010). Assessing the Role of Spin Noise in the Precision Timing of Millisecond Pulsars, ApJ 725, pp. 1607-1619.

118. Shannon, R. M., Osłowski, S., Dai, S., Bailes, M., Hobbs, G., Manchester, R. N., van Straten, W., Raithel, C. A., et al. (2014). Limitations in timing precision due to single-pulse shape variability in millisecond pulsars, MNRAS 443, pp. 1463-1481.

119. Shannon, R. M., Ravi, V., Coles, W. A., Hobbs, G., Keith, M. J., Manchester, R. N., Wyithe, J. S. B., Bailes, M., et al. (2013). Gravitational-wave limits from pulsar timing constrain supermassive black hole evolution., Science 342, pp. 334-337.

120. Shao, L., Caballero, R. N., Kramer, M., Wex, N., Champion, D. J. and Jessner, A. (2013). A new limit on local Lorentz invariance violation of gravity from solitary pulsars, Class. Quant. Grav. 30, 16, 165019.

121. Shao, L. and Wex, N. (2012). New tests of local Lorentz invariance of gravity with small-eccentricity binary pulsars, Class. Quant. Grav. 29, 21, p. 215018.

122. Shao, L. and Wex, N. (2013). New limits on the violation of local position invariance of gravity, Class. Quant. Grav. 30, 16, 165020.

123. Shapiro, I. I. (1964). Fourth test of general relativity, Phys. Rev. Lett. 13, p. 789.

124. Shen, Y., Liu, X., Loeb, A. and Tremaine, S. (2013). Constraining Sub-parsec Binary Supermassive Black Holes in Quasars with Multi-epoch Spectroscopy. I. The General Quasar Population, ApJ 775, 49.

125. Shklovskii, I. S. (1970). Possible causes of the secular increase in pulsar periods, Sov. Astron. 13, pp. 562-565.

126. Siemens, X., Ellis, J., Jenet, F. and Romano, J. D. (2013). The stochastic background: scaling laws and time to detection for pulsar timing arrays, Class. Quant. Grav. 30, $22,224015$.

127. Siemens, X., Mandic, V. and Creighton, J. (2007). Gravitational-Wave Stochastic Background from Cosmic Strings Phys. Rev. D 98, 11, p. 111101.

128. Springel, V., White, S. D. M., Jenkins, A., Frenk, C. S., Yoshida, N., Gao, L., Navarro, J., Thacker, R., et al. (2005). Simulations of the formation, evolution and clustering of galaxies and quasars, Nature 435, pp. 629-636.

129. Stairs, I. H. (2003). Testing general relativity with pulsar timing, Living Rev. Relativity 6, URL (accessed November 2014): http://relativity.livingreviews.org/Articles/lrr-2003-5.

130. Stairs, I. H., Faulkner, A. J., Lyne, A. G., Kramer, M., Lorimer, D. R., McLaughlin, M. A., Manchester, R. N., Hobbs, G. B., et al. (2005). Discovery of three wide-orbit binary pulsars: implications for binary evolution and equivalence principles, $A p J$ 632, pp. 1060-1068.

131. Stairs, I. H., Thorsett, S. E., Taylor, J. H. and Wolszczan, A. (2002). Studies of the 
relativistic binary pulsar PSR B1534+12: I. timing analysis, ApJ 581, pp. 501-508.

132. Stewart, K. R., Bullock, J. S., Barton, E. J. and Wechsler, R. H. (2009). Galaxy Mergers and Dark Matter Halo Mergers in $\Lambda$ CDM: Mass, Redshift, and Mass-Ratio Dependence, ApJ 702, pp. 1005-1015.

133. Sudou, H., Iguchi, S., Murata, Y. and Taniguchi, Y. (2003). Orbital Motion in the Radio Galaxy 3C 66B: Evidence for a Supermassive Black Hole Binary, Science 300, pp. $1263-1265$.

134. Taylor, J. H., Fowler, L. A. and McCulloch, P. M. (1979). Measurements of general relativistic effects in the binary pulsar PSR 1913+16, Nature 277, p. 437.

135. Taylor, J. H., Hulse, R. A., Fowler, L. A., Gullahorn, G. E. and Rankin, J. M. (1976). Further observations of the binary pulsar PSR 1913+16, ApJ 206, pp. L53-L58.

136. Thorsett, S. E. (1996). The gravitational constant, the Chandrasekhar limit, and neutron star masses, Phys. Rev. Lett. 77, p. 1432.

137. Valtonen, M. J., Lehto, H. J., Takalo, L. O. and Sillanpää, A. (2011). Testing the 1995 Binary Black Hole Model of OJ287 ApJ 729, 33.

138. van Haasteren, R., Levin, Y., Janssen, G. H., Lazaridis, K., Kramer, M., Stappers, B. W., Desvignes, G., Purver, M. B., et al. (2011). Placing limits on the stochastic gravitational-wave background using European Pulsar Timing Array data, MNRAS 414, pp. 3117-3128.

139. van Leeuwen, J., Kasian, L., Stairs, I. H., Lorimer, D. R., Camilo, F., Chatterjee, S., Cognard, I., Desvignes, G., et al. (2015). The Binary Companion of Young, Relativistic Pulsar J1906+0746, ApJ 798, 118.

140. Venemans, B. P., Findlay, J. R., Sutherland, W. J., De Rosa, G., McMahon, R. G., Simcoe, R., González-Solares, E. A., Kuijken, K. and Lewis, J. R. (2013). Discovery of Three $\mathrm{z}>6.5$ Quasars in the VISTA Kilo-Degree Infrared Galaxy (VIKING) Survey, ApJ 779, 24.

141. Verbiest, J. P. W., Bailes, M., van Straten, W., Hobbs, G. B., Edwards, R. T., Manchester, R. N., Bhat, N. D. R., Sarkissian, J. M., et al. (2008). Precision timing of PSR J0437-4715: an accurate pulsar distance, a high pulsar mass and a limit on the variation of Newton's gravitational constant, ApJ 679, pp. 675-680.

142. Vilenkin, A. (1981). Gravitational radiation from cosmic strings, Phys Lett B 107, pp. $47-50$.

143. Weisberg, J. M., Nice, D. J. and Taylor, J. H. (2010). Timing Measurements of the Relativistic Binary Pulsar PSR B1913+16, ApJ 722, pp. 1030-1034.

144. Weisberg, J. M., Romani, R. W. and Taylor, J. H. (1989). Evidence for geodetic spin precession in the binary pulsar 1913+16, ApJ 347, pp. 1030-1033.

145. Weisberg, J. M. and Taylor, J. H. (2002). General Relativistic Geodetic Spin Precession in Binary Pulsar B1913+16: Mapping the Emission Beam in Two Dimensions, ApJ 576, pp. 942-949.

146. Wex, N. (2014). Testing Relativistic Gravity with Radio Pulsars, ArXiv e-prints To appear in the Brumberg Festschrift (ed. S. M. Kopeikein), de Gruyter, Berlin.

147. Will, C. M. (1992). Is momentum conserved? A test in the binary system PSR 1913+16, ApJ 393, pp. L59-L61.

148. Will, C. M. (1993). Theory and Experiment in Gravitational Physics (Cambridge University Press, Cambridge).

149. Will, C. M. (2014). The Confrontation between General Relativity and Experiment, Living Rev. Relativity 17, p. 4.

150. Will, C. M. and Nordtvedt, K. J. (1972). Conservation Laws and Preferred Frames in Relativistic Gravity. I. Preferred-Frame Theories and an Extended PPN Formalism, ApJ 177, pp. 757-774. 
151. Williams, J. G., Turyshev, S. G. and Boggs, D. H. (2012). Lunar laser ranging tests of the equivalence principle, Class. Quant. Grav. 29, 18, 184004.

152. Wolszczan, A. (1991). A nearby $37.9 \mathrm{~ms}$ radio pulsar in a relativistic binary system, Nature 350, pp. 688-690.

153. Wolszczan, A., Doroshenko, O., Konacki, M., Kramer, M., Jessner, A., Wielebinski, R., Camilo, F., Nice, D. J. and Taylor, J. H. (2000). Timing observations of four millisecond pulsars with the Arecibo and Effelsberg radio telescopes, ApJ 528, pp. 907-912.

154. Wyithe, J. S. B. and Loeb, A. (2003). Low-Frequency Gravitational Waves from Massive Black Hole Binaries: Predictions for LISA and Pulsar Timing Arrays, ApJ 590, pp. 691-706.

155. Yagi, K., Blas, D., Barausse, E. and Yunes, N. (2014). Constraints on EinsteinÆther theory and Hořava gravity from binary pulsar observations, Phys. Rev. D 89, 8, 084067.

156. Yan, W. M., Manchester, R. N., van Straten, W., Reynolds, J. E., Hobbs, G., Wang, N., Bailes, M., Bhat, N. D. R., et al. (2011). Polarization observations of 20 millisecond pulsars, MNRAS 414, pp. 2087-2100.

157. Zhang, X.-G., Dultzin-Hacyan, D. and Wang, T.-G. (2007). SDSS J1130+0058 an $\mathrm{X}$-shaped radio source with double-peaked low-ionization emission lines: a binary black hole system?, MNRAS 377, pp. 1215-1221.

158. Zhu, X.-J., Hobbs, G., Wen, L., Coles, W. A., Wang, J.-B., Shannon, R. M., Manchester, R. N., Bailes, M., et al. (2014). An all-sky search for continuous gravitational waves in the Parkes Pulsar Timing Array data set, MNRAS 444, pp. 3709-3720. 